\title{
Molecular Dynamics Simulation of Self-assembly of n-Decyltrimethylammonium Bromide Micelles
}

\author{
Miguel Jorge* \\ Laboratory of Separation and Reaction Engineering (LSRE), Faculdade de Engenharia, \\ Universidade do Porto, Rua Dr. Roberto Frias s/n, 4200-465 Porto, Portugal \\ and \\ REQUIMTE, Faculdade de Ciências, Universidade do Porto, Rua do Campo Alegre, 687, 4169- \\ 007 Porto, Portugal \\ Email address: mjorge@fe.up.pt
}

Title Running Head: Self-assembly of DeTAB micelles

\begin{abstract}
In this paper, a molecular dynamics simulation of surfactant self-assembly using realistic atomistic models is presented. The simulations are long enough to enable the observation of several processes leading to equilibrium, such as monomer addition and detachment, micelle dissolution, and micelle fusion. The self-assembly of DeTAB surfactants takes place in three stages: fast aggregation of monomers to form small disordered oligomers; ripening process by which larger aggregates grow at the expense of smaller ones; slower stage involving collisions
\end{abstract}


between large micelles. The first two stages were well described by a simple kinetic model with a size-independent rate constant estimated from the self-diffusion coefficient and collision radius of an isolated monomer. The average cluster size, area per head-group, degree of counterion dissociation and critical micelle concentration estimated from the simulation are in reasonable agreement with experimental values. An all-atom and a united-atom surfactant model were compared, and the results were seen to be almost independent of the choice of model. DeTAB micelles are spheroidal, with a hydrophobic core composed of tail atoms surrounded by a hydrophilic corona of head atoms. A Stern layer composed of bromide counterions was also identified. Water molecules solvate the counterions and the head atoms, penetrating into the micelle up to the location of the atom connecting the head to the aliphatic tail, in agreement with recent experimental observations.

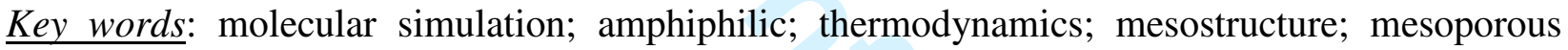
silica.

\section{Introduction}

Surfactant molecules are important in a wide variety of processes: biological, as in molecule transport across cell membranes; commercial, as in detergents and stain removers; and industrial, as in emulsion polymerization. Their unique properties stem from their amphiphilic nature, i.e., each molecule contains both a hydrophilic and a hydrophobic section. When dissolved in water above a certain concentration, termed the critical micelle concentration $(\mathrm{cmc})$, surfactants self-assemble into mesoscopic aggregates, such as micelles, vesicles and bilayers. The precise structure of these aggregates depends on the type and concentration of surfactant, temperature, presence of co-solvent or dissolved electrolytes and other factors, giving rise to extremely rich phase diagrams ${ }^{1}$. Thus, not surprisingly, they have been the subject of numerous 
experimental $^{2}$ and theoretical ${ }^{3}$ studies, which for the most part focused on phase equilibrium and, more recently, on the investigation of aggregate structure. One aspect that has so far eluded experimental probing is the actual mechanism of surfactant self-assembly, due to the short time and length scales over which it takes place. In this paper, we attempt to shed light on this issue by applying molecular dynamics (MD) to explicitly simulate the self-assembly of ndecyltrimethylammonium bromide (DeTAB) surfactants into multiple micellar aggregates.

Molecular simulation methods have been quite successful in complementing experimental techniques in the study of surfactant solutions. A great deal of insight about the structure of micellar aggregates and their interactions with the solvent was obtained from molecular simulations starting from pre-formed micelles. Indeed, one such study has been performed on an n-decyltrimethylammonium chloride micelle composed of 30 surfactants in water ${ }^{4}$ and another on a solvated DeTAB micelle with 47 surfactants $^{5}$. However, because they focus on a single equilibrated micelle, these studies yield no information about the process by which the aggregate is formed. Due to limitations in computer power, early simulations of the dynamics of micelle formation had to rely on mesoscopic methods (such as lattice-gas and dissipative particle dynamics $)^{6,7}$ or on coarse-grained models of surfactants, either in continuum ${ }^{8,9}$ or on a lattice ${ }^{10,11}$. These methods are useful for describing general phenomena involved in self-assembly but their main shortcoming is the difficulty in establishing quantitative links to real systems ${ }^{3}$.

To our knowledge, the first explicit simulations of surfactant aggregation using realistic models were performed in 1998. Tarek et al. ${ }^{12}$ performed a $4.5 \mathrm{~ns}$ MD simulation starting from a random solution of 42 sodium dodecyl sulfate molecules, and were able to observe the formation of two small aggregates. Soon after, Maillet et al. ${ }^{13}$ studied the self-assembly of a short-chain surfactant (n-nonyltrimethylammonium chloride) and a long-chain surfactant (erucyl bis[2hydroxyethyl]methylammonium chloride) in water, using systems containing about 50 
amphiphiles for 3 ns. During the short-chain surfactant simulations, they have observed micelle formation, micelle fragmentation and monomer exchange with the surrounding solution, resulting in systems with two approximately spherical micelles. Based on their observations, they proposed that the initial stages of the process are dominated by collisions between aggregates, while the later stages, closer to equilibrium, proceed mostly by monomer exchanges. Salaniwal et al. ${ }^{14}$ simulated the formation of reverse micelles in a solution of water, surfactant and supercritical $\mathrm{CO}_{2}$. Their system had 30 surfactant molecules and ran for a little over $1 \mathrm{~ns}$. Nevertheless, three small stable aggregates were formed during this short time, due to the rapid monomer exchange dynamics in supercritical $\mathrm{CO}_{2}$. Later, Marrink et al. ${ }^{15}$, studied by MD the self-assembly of 54 dodecylphosphocholine molecules for a significantly longer period, $18 \mathrm{~ns}$, and compared the simulated rate of aggregation to predictions from kinetic theory. At high concentration, the surfactants aggregated into a wormlike micelle, while a single spherical micelle was the outcome at low concentration.

The studies mentioned above have been restricted to small systems (around 50 surfactants) and, except for that of Marrink et al. ${ }^{15}$, to short simulation times, reflecting the computational resources available at the time. In this paper, we use high-performance parallel computers to examine the self-assembly process of DeTAB micelles using realistic atomistic models and long simulation times. We also compare the performance of two surfactant models, one based on an all-atom description (including explicit hydrogens) and the other - faster but less rigorous - based on a united-atom approach. The paper is organized as follows: in section 2 we give details about the computational methods; in section 3 we present our results, focusing first on describing the self-assembly process and then on the structures of the resulting micellar aggregates; finally, we present our conclusions in section 4. 


\section{Computational Details}

All simulations were performed using version 3.3 of the GROMACS ${ }^{16,17}$ MD simulation package. Equations of motion were integrated with the Verlet leapfrog algorithm ${ }^{18}$ and a time step of 2 fs. Simulations were carried out in the $N p T$ ensemble - the temperature was kept fixed at $353.15 \mathrm{~K}$ by applying the Nosé-Hoover thermostat ${ }^{19,20}$, while the pressure was held constant at 1 bar using the Parrinello-Rahman barostat ${ }^{21}$. We chose a temperature higher than room temperature (at which most self-assembly experiments are performed) in order to increase the mobility of surfactant molecules, thus speeding up the aggregation process. The simulation box was cubic, with periodic boundary conditions in all Cartesian directions.

Water molecules were represented by the SPC/E potential ${ }^{22}$ with rigid bonds and bond angle. Two models were used for the DeTAB surfactants: an all-atom (AA) model and a unitedatom (UA) model. The main difference between the models is that the AA model includes explicit hydrogen atoms, while the UA model represents each $\mathrm{CH}_{3}$ and $\mathrm{CH}_{2}$ group by a single effective interaction center. The parameters for the AA model were taken from the OPLS potential $^{23}$. In the case of the UA model, parameters for the head group were taken from Jorgensen and $\mathrm{Gao}^{24}$ and parameters for the aliphatic tail were taken from Smit et al. ${ }^{25}$. In both models, the bond lengths were kept rigid, with constraints enforced using the LINCS algorithm ${ }^{26}$. The total potential energy is thus the sum of angle-bending terms, torsional terms, Lennard-Jones and Coulomb electrostatic terms. A twin-range cutoff scheme was employed to calculate the short-range dispersion interactions, with cutoff radii of 1.0 and $1.2 \mathrm{~nm}$. This means that interactions between atom pairs separated by less than $1.0 \mathrm{~nm}$ are computed at every time step, while those between atoms separated by more than $1.0 \mathrm{~nm}$ and less than $1.2 \mathrm{~nm}$ are also added at every time step, but their value is only updated at every 10 time steps. Interactions involving atoms separated by more than $1.2 \mathrm{~nm}$ are included in a long-range dispersion correction term, 
added to both energy and pressure. Long-range electrostatics were handled using the particlemesh Ewald method ${ }^{27}$ with a real-space cutoff of $1.0 \mathrm{~nm}$. The angle-bending potential $\left(U_{\theta}\right)$ was given by a harmonic function of the form:

$$
U_{\theta}=\frac{1}{2} k_{\theta}\left(\theta-\theta_{0}\right)^{2}
$$

where $\theta$ is the instantaneous value of the angle, $\theta_{0}$ is the equilibrium angle and $k_{\theta}$ is the force constant. For the dihedral potential $\left(U_{\varphi}\right)$, we used the Ryckaert-Bellemans form:

$$
U_{\varphi}=\sum_{n=0}^{5} C_{\mathrm{n}}(\cos (\varphi))^{n}
$$

where $\varphi$ is the dihedral angle and $C_{\mathrm{n}}$ is the coefficient of order $n$ in the expansion. In the particular case of the potentials employed in this work, coefficients $C_{4}$ and $C_{5}$ were always equal to zero. The full set of potential parameters for both surfactant models is given in Tables 1 to 4 , and a schematic diagram of a $\mathrm{DeTA}^{+}$cation, with the identification of each atom type, is shown in Figure 1.

\section{Figure 1}

\section{Table 1}

Table 2

Table 3

\section{Table 4}

The starting configurations were constructed as follows. First, a small cubic box containing one $\mathrm{DeTA}^{+}$cation and one bromide counterion solvated in 50 water molecules was constructed and equilibrated. The equilibration stage consisted of a steepest descent energy minimization followed by a short MD run in the $N p T$ ensemble. Due to the small size of this box, the cutoff radius was reduced to $0.6 \mathrm{~nm}$, so that it was always less than half of the box length. 
Once equilibrated, the small box was replicated in a cubic array, 5 times in each Cartesian direction. Thus, we obtained a large box containing 125 DeTAB pairs and 6250 water molecules, with an average surfactant concentration of $0.83 \mathrm{~mol} / \mathrm{l}$. After a short energy minimization to eliminate short-range contacts between atoms, the MD production runs were started. For each model, a run of $14 \mathrm{~ns}$ was performed, with coordinates saved at every $0.2 \mathrm{ps}$. In addition to the large simulations of self-assembly, we have performed simulations of a single DeTAB ion pair solvated in a periodic cubic box with 280 water molecules. For each surfactant model, one MD run of $10 \mathrm{~ns}$ was carried out in order to calculate properties of isolated surfactants.

An important part of the analysis is the identification of surfactant aggregates in each sampled configuration. For that purpose, one must establish a criterion to determine whether two surfactant molecules belong to the same aggregate. We have tested two different types of criterion, one specifying a threshold distance between the center of mass (COM) of each molecule, and another based on a threshold distance between surfactant tail carbon atoms. In the latter case, different combinations of tail atoms were tested. For all tests, the identification and classification of the aggregates was carried out using an adaptation of the Hoshen-Kopelman cluster-counting algorithm ${ }^{28}$. In each case, the results of the algorithm were tested by visual inspection of the configurations. The best criterion, providing a good balance between accuracy, robustness and speed of computation, considers that two surfactant molecules belong to the same aggregate if any of their last 4 tail atoms are separated by no more than $0.64 \mathrm{~nm}$. In fact, the results were essentially the same provided that the threshold distance was between 0.56 and 0.72 $\mathrm{nm}$. These values are close to the position of the first minimum in the radial distribution functions between tail carbon atoms. Considering more tail atoms increased the computation time without improving the accuracy, while considering fewer tail atoms significantly reduced the accuracy and robustness of the procedure (it became much more sensitive to the threshold distance). The 
criterion based on the surfactant COM was somewhat faster than the best criterion, but the results were very sensitive to the threshold distance (it had to be between 0.92 and $0.94 \mathrm{~nm}$ to yield accurate results).

\section{Results and Discussion}

\subsection{Self-assembly Process}

We begin by analyzing in detail the process by which surfactant molecules cluster together to form a number of micellar aggregates. Figure 2 shows the evolution in time of the mass-average cluster size during the entire simulation run, and of the total number of aggregates in the simulation box during the first $500 \mathrm{ps}$ of the simulation. It should be noted that the total number of aggregates takes into account all clusters, including monomers and small oligomers, while the calculation of the average cluster size includes only aggregates with 5 or more surfactant molecules (which we define to be the smallest aggregate which counts as a micelle). Almost as soon as the simulation begins, surfactant molecules start to aggregate. The total number of aggregates thus decreases very rapidly during the early stages of the run (Figure $2 \mathrm{~b}$ ). This decrease slows down with time, and beyond about 1000 ps major changes in the average cluster size occur only by virtue of occasional jumps (Figure 2a). During the last 7 ns of both simulation runs, the mass-average cluster size oscillates around a fairly constant value, between 22 and 27 surfactant molecules.

\section{Figure 2}

Another important observation coming from Figure 2 is that the mechanism of selfassembly seems to be unaffected by the choice of surfactant model. Indeed, the curves for the AA and UA models show similar behavior, with two minor differences: a slightly larger final massaverage cluster size for the UA model (Figure 2a) and an extremely fast initial decrease in the 
number of clusters for the AA model (Figure 2b). A close analysis of the first few ps of simulation reveals the reason for the latter. The construction procedure described in section 2 produces large cubic boxes with volumes close to, but not exactly at equilibrium. This is due to the fact that the small box was equilibrated using a significantly smaller cutoff radius $(0.6 \mathrm{~nm})$ than the one used in the large box $(1.2 \mathrm{~nm})$, which means that the density of the large box will not be precisely the same as that of the small box. As such, during a very short period at the beginning of the run, the enforced pressure-coupling will drive the system toward its equilibrium volume. During this approach to equilibrium, the Parrinello-Rahman barostat typically produces an oscillatory response in the system volume, the amplitude of which is related to the chosen value for the coupling constant. In the simulation with the UA model, the initial oscillation leading to equilibrium was of the same magnitude as typical volume oscillations at equilibrium. Thus, the system evolved smoothly in time. In the AA simulation, however, a much larger volume oscillation took place during the first $3.5 \mathrm{ps}$. This caused a short-lived decrease in the system density, substantially increasing the mobility of surfactant molecules. As we will discuss below, the rate of aggregation in the initial stages of the simulation depends strongly on the probability of contact between surfactant monomers, and will therefore be related to their selfdiffusion coefficient. Thus, the result of this momentary increase in mobility was a sudden drop in the total number of clusters, observed in Figure $2 \mathrm{~b}$ at very short times. After this short period, the self-assembly proceeded normally. In principle, it might be possible to minimize this artifact by decreasing the coupling constant of the barostat (thus imposing smaller oscillations), or by employing a different procedure to build the starting configuration. However, this was not attempted here since the effect was only detected after all the simulation runs were concluded.

We have performed a more detailed analysis of the self-assembly process by monitoring the evolution of each individual aggregate in time. During the short period of time between 
successive sampled configurations, the aggregate center of mass changes only slightly. The cluster may also grow by colliding with another aggregate or shrink by shedding part of its mass. In each configuration, we have identified each aggregate and calculated its size and center of mass. We track each aggregate from one configuration to the next by finding the cluster whose $\mathrm{COM}$ is closest to the $\mathrm{COM}$ position of a given cluster in the previous configuration. This simple tracking method works well provided that the time interval between successive configurations is not too large. The effectiveness of the method was verified by visual inspection of the data in randomly chosen pairs of consecutive configurations.

The results of the tracking procedure at early stages of the simulation runs are very difficult to interpret, due to the large number of aggregates present in the box. Nevertheless, they allow us to observe that the initial stage of cluster growth (in the first tens of picoseconds) proceeds by quick aggregation of individual monomers to form small clusters. In Figure 3a, we show the cluster size distribution (CSD) for the UA model obtained by averaging over all configurations between 60 and 100 ps of simulation time. One can see that the system is composed mainly of monomers and small oligomers (up to 5 molecules), with also a few intermediate-sized clusters present. Visual inspection of simulation snapshots, such as those shown in Figure 4, indicates that the oligomers have fairly disordered structures. These clusters are rather unstable, and quickly dissolve, providing matter for the growth of more stable, intermediate-sized clusters. This trend is evident in the CSDs shown in Figure 3: as the simulation progresses, the shoulder in the distribution corresponding to small oligomers virtually disappears, while clusters formed by 10 to 20 molecules become predominant. Analysis of the cluster tracking data in this region confirms that the cluster growth proceeds mainly by monomer addition, with less frequent collisions involving small oligomers. This growth process is an example of Ostwald ripening, by which smaller particles dissolve and provide material for the 
growth of larger, more stable, particles ${ }^{29}$. After about 700 ps of simulation time, the ripening process is practically concluded - the system is now composed almost exclusively of monomers and intermediate-sized micelles (see Figure 3d).

\section{Figure 3}

\section{Figure 4}

MD simulations provide information about the real-time dynamics of the model system. That information may be used, for instance, to test kinetic models of aggregation. Marrink et al. ${ }^{15}$ have compared their simulation data on the self-assembly of dodecylphosphocholine micelles to theoretically predicted aggregation rates. Their models ranged from a simplified analytical expression based on a size-independent rate constant to a more complex stochastic approach, but they were unable to obtain good agreement between simulation and theory. In this paper, we compare our simulation data to the simplified model derived by Marrink et al. ${ }^{15}$, together with a rough estimate of the size-independent rate constant $(k)$. We begin by describing the selfassembly process, to first order, by the following reaction:

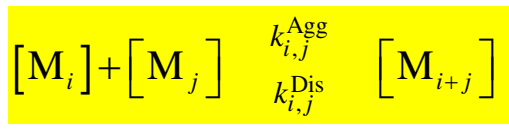

where $\left[\mathrm{M}_{i}\right]$ represents the concentration of clusters with $i$ surfactant molecules. The rate constants may depend on the size of both colliding clusters (subscript $i, j$ ). Superscripts Agg and Dis are for the aggregation and dissociation processes, respectively. During the early stages of the approach to equilibrium, it is reasonable to assume that the dissociation process is much slower than the aggregation process, and it is thus possible to neglect the former ${ }^{15}$. Based on this assumption, a mass balance on clusters of size $i$ yields the following rate equation for cluster aggregation:

$$
\frac{\mathrm{d}\left[\mathrm{M}_{i}\right]}{\mathrm{d} t}=\sum_{j=1}^{i / 2} k_{i-j, j}\left[\mathrm{M}_{i-j}\right]\left[\mathrm{M}_{j}\right]-\left[\mathrm{M}_{i}\right] \sum_{j=1} k_{i, j}\left[\mathrm{M}_{j}\right]-k_{i, i}\left[\mathrm{M}_{i}\right]^{2}
$$


where we have dropped the superscript Agg for simplicity of notation. The first term on the righthand side represents the formation of clusters of size $i$ due to the collision of two smaller clusters, while the second and third terms describe the disappearance of clusters of size $i$ due to collisions with other clusters. The initial conditions are that at the start of the self-assembly process there are only isolated monomers in solution (i.e., for $t=0,\left[M_{1}\right]=\mathrm{M}_{0}$ and $\left[M_{\mathrm{i}>1}\right]=0 ; \mathrm{M}_{0}$ is the initial surfactant concentration). If one assumes a size-independent rate constant, equation (4) may be solved analytically with the above initial conditions, giving rise to the following general solution:

$$
\left[\mathrm{M}_{n}\right]=\mathrm{M}_{0}\left(\frac{t}{\tau}\right)^{n-1}\left(1+\frac{t}{\tau}\right)^{-n-1}
$$

with $\tau=2 / k \mathrm{M}_{0}$. The concentration of isolated monomers in solution as a function of time follows directly from equation (5) with $n=1$ :

$$
\left[\mathrm{M}_{1}\right]=\mathrm{M}_{0}\left(1+\frac{t}{\tau}\right)^{-2}
$$

One may also calculate the time dependence of the total concentration of clusters by summing equation (5) over all cluster sizes:

$$
\left[\mathrm{M}_{\mathrm{tot}}\right]=\sum_{i=1}^{\infty}\left[\mathrm{M}_{i}\right]=\mathrm{M}_{0}\left(1+\frac{t}{\tau}\right)^{-1}
$$

Finally, one can also obtain an expression for the average cluster size as a function of time. The general expression for the number-average cluster size $\left(C S_{\mathrm{N}}\right)$ is:

$$
\left\langle C S_{\mathrm{N}}\right\rangle_{i}=\frac{\sum_{n=i}^{\infty} n\left[\mathrm{M}_{n}\right]}{\sum_{n=i}^{\infty}\left[\mathrm{M}_{n}\right]}
$$

In the above averaging procedure, we count all clusters that are larger than $i$. If we consider all clusters, including monomers (i.e., $i=1$ ), then $C S_{\mathrm{N}}$ is simply the starting surfactant concentration $\left(\mathrm{M}_{0}\right)$ divided by the total concentration of clusters, given by equation (7). In our analysis, 
however, we are interested in the average micelle size, without taking isolated monomers and small oligomers into account. Thus, we calculate the average size of clusters that have at least 5 surfactant molecules. Substituting equation (5) in equation (8) with $i=5$ we obtain, after some algebra:

$$
\left\langle C S_{\mathrm{N}}\right\rangle_{5}=\frac{\left(1+\frac{t}{\tau}\right)^{5}-\left(1+\frac{t}{\tau}\right)^{3}-2\left(\frac{t}{\tau}\right)\left(1+\frac{t}{\tau}\right)^{2}-3\left(\frac{t}{\tau}\right)^{2}\left(1+\frac{t}{\tau}\right)-4\left(\frac{t}{\tau}\right)^{3}}{\left(\frac{t}{\tau}\right)\left(1+\frac{t}{\tau}\right)^{3}-\left(\frac{t}{\tau}\right)\left(1+\frac{t}{\tau}\right)^{2}-\left(\frac{t}{\tau}\right)^{2}\left(1+\frac{t}{\tau}\right)-\left(\frac{t}{\tau}\right)^{3}}
$$

To compare our simulation data to the predictions based on the above set of equations, we need an estimate for the size-independent rate constant. Once again, we follow Marrink et al. ${ }^{15}$ and start from the expression for diffusion-controlled aggregation:

$$
k_{i, j}=4 \pi\left(D_{i}+D_{j}\right)\left(R_{i}+R_{j}\right)
$$

where, for a cluster with $i$ surfactants, $D_{i}$ is its diffusion coefficient and $R_{i}$ is its collision radius. Since we are assuming a size-independent $k$, equation (10) becomes simply:

$$
k=16 \pi D R
$$

Furthermore, the initial surfactant concentration is given by:

$$
\mathrm{M}_{0}=\frac{\mathrm{N}_{0}}{L^{3}}
$$

where $\mathrm{N}_{0}$ is the initial number of surfactant molecules and $L$ is the length of each side of the simulation box. Using equations (11) and (12), we obtain the following expression for the characteristic aggregation time:

$$
\tau=\frac{L^{3}}{8 \pi D R \mathrm{~N}_{0}}
$$

Our previous observations have shown that the early stages of self-assembly proceed almost exclusively by collisions involving monomers and small oligomers. Therefore, as a first 
estimate, we have used the diffusion coefficient and the collision radius of an isolated surfactant monomer in equation (13). These properties were computed from simulations of single DeTAB ion pairs dissolved in water, described in section 2. $D$ was calculated from the slope of the mean square displacement of $\operatorname{DeTA}^{+}$cations, according to the Einstein relation ${ }^{30}$, while $R$ was estimated as half of the average distance between the last tail carbon atom (MT in Figure 1) and each of the three carbon atoms of the head methyl groups ( $\mathrm{MH}$ in Figure 1). Furthermore, because the box volume was allowed to vary during the simulations (see section 2), the average value of the box length for each run was used in equation (13). Table 5 lists the average values of $D, R$ and $L$, as well as the calculated values of $\tau$ for both the UA and the AA models.

\section{Table 5}

The diffusion coefficients calculated from the simulations of both model surfactants may be compared to experimental determinations of this property. However, experimental values for diffusion of $\mathrm{DeTA}^{+}$cations in water are available at room temperature $(298 \mathrm{~K})$ rather than the temperature at which we are studying aggregation $(353 \mathrm{~K})$. Therefore, in order to validate our results, we have performed simulations of isolated surfactants in water at the lower temperature. The calculated values of $D$ at $298 \mathrm{~K}$, also shown in Table 5, compare well with the experimental results of D'Errico et al. ${ }^{31}\left(0.636\right.$ to $\left.0.667 \times 10^{9} \mathrm{~m}^{2} / \mathrm{s}\right)$ and of Ribeiro et al. ${ }^{32}\left(0.726 \times 10^{9} \mathrm{~m}^{2} / \mathrm{s}\right)$, with slightly better agreement for the AA model. Thus, we may conclude that both models provide a good description of the dynamic behavior of isolated surfactant molecules and that this description is slightly better for the more realistic AA model.

With our estimates of $\tau$, we are now in a position to compare the predictions of equations (6), (7) and (9) to the simulation results. Figure 5 shows such a comparison for both surfactant models. Two issues should be noted regarding this figure: (i) for clarity, we have presented the results and predictions in terms of the number of monomers and clusters, rather than their 
concentration; (ii) for the AA model, we have disregarded the short initial period corresponding to equilibration of the box volume, during which molecular mobility was abnormally increased (see discussion above), and started our predictions from the values of $\mathrm{M}_{1}$ and $\mathrm{M}_{\mathrm{tot}}$ observed at $t=$ 3.5 ps. It becomes immediately clear that the kinetic model derived above is able to faithfully describe the evolution of the system during the first 700 ps of simulation time. This is remarkable, given that the model is extremely simplified and our estimate of $k$ is rather crude. Indeed, the model is strictly valid only for an infinite system with homogeneously distributed clusters, thus neglecting correlations and thermodynamic fluctuations, and does not take into account potential interactions between clusters ${ }^{15}$. The excellent performance of the kinetic model thus suggests that the early stages of self-assembly are dominated by diffusion-controlled aggregation of monomers and small oligomers, in full agreement with our qualitative observations.

\section{Figure 5}

After about 700 ps, the mechanism of cluster growth changes, and the simple kinetic model is no longer able to describe the simulation data. Beyond the first stage, growth or shrinkage of individual micelles by monomer addition or dissolution still takes place very frequently, but the overall impact on the average cluster size is minimal. In fact, the size of most of these micelles changes very little over long time spans, simply fluctuating around an average value. In this later stage, the average cluster size increases mainly by occasional collision events involving relatively large micelles. These collisions manifest themselves as jumps in the plot of Figure 2a. In Figure 6, we present snapshots obtained from the UA simulation run over a time period during which an event of micelle fusion takes place. In this figure, only the colliding micelles are shown, and surrounding water molecules are removed for clarity. Figure 7 shows the results of tracking these two micelles over the time period of the fusion event. Initially, both 
micelles are isolated (Figure 6a), with distinct coronae and associated counterion clouds, separated by a significant water layer. At this stage, the micelles have similar COM coordinates in the $x$ and $y$ directions (dotted and dashed lines, respectively, in Figure 7b) but are far apart in the $z$ direction (full lines in Figure 7b). The smallest micelle has about 12 surfactant molecules, while the largest one has about 16 surfactants (see Figure 7a). After about 2 ns, the micelles move closer together (Figure 6b) and their counterion clouds begin to overlap. Bromide ions are strongly bound to the micelle head groups (see discussion in section 3.2) and effectively screen the positive charge at the micelle surface. This screening reduces the mutual repulsion between individual micelles, and facilitates their collision. Without the presence of the counterions, the electrostatic repulsion between micelles would most likely make any fusion events prohibitive.

After a very short period, the head-group regions of the micelles begin to interpenetrate (Figure 6c). Due to mutual repulsions, the head groups that were located in the contact region begin to move toward the periphery. At $t=4360 \mathrm{ps}$, tail atoms of the two micelles make contact (Figure 6d), and the cluster-counting algorithm considers that they form a single aggregate. The plots in Figure 7 show this fusion, which causes the formation of a micelle containing 28 surfactants. After the fusion, the surfactant molecules in the large micelle quickly rearrange themselves so that all head atoms are displaced to the surface of the aggregate. After about another 200 ps (Figure 6e), the micelle exhibits an equilibrated structure.

\section{Figure 6}

\section{Figure 7}

In summary, the self-assembly of DeTAB micelles under the conditions studied takes place in several stages. In our simulations we are able to identify: a fast monomer aggregation process, forming small aggregates; a "ripening" stage, during which small unstable clusters dissolve forming more stable intermediate-sized micelles; and a slow stage during which growth 
proceeds primarily by collisions between large micelles, the size of which fluctuates via monomer exchanges. Our DeTAB solutions are quite concentrated, and so micelle collisions are relatively frequent in the first 5 ns. Beyond this time, however, collisions become rare, presumably because the micelles are now quite stable and far apart from each other. This leads to the long plateaus observed in Figure 2a. In a more dilute system and/or at lower temperature, micelle collisions will be less frequent (in the first case, due to larger separation distances and in the second case, due to lower mobility), and longer simulations will be necessary to reach equilibrium.

\subsection{Micellar Properties}

In this section, we report properties of the micellar systems averaged over the last $7 \mathrm{~ns}$ of each simulation run. During this period, the average cluster size shows a steady plateau and oscillates around an average value (see Figure 2a). In Figure 8 we show the average cluster size distributions calculated during this period. Both distributions are rather spiky, due to poor statistics - typical configurations contain between 8 and 12 individual micelles, which is a large number compared to previous atomistic simulations, but still too small to obtain smooth CSDs. Importantly, we can clearly distinguish a trough between the peak for monomers and small oligomers (up to about 5 surfactant molecules) and the set of peaks for micelles (spread between 10 and 33 surfactants). Simulations of coarse-grained models, which are able to span larger length scales and longer times, have produced CSDs that are qualitatively similar to our own, but with a single smooth peak for micelles ${ }^{8,11}$.

\section{Figure 8}

Another conclusion that can be drawn from Figure 8 is that the CSDs for both surfactant models are qualitatively similar. The same can be said of the average cluster size for each model. 
In Table 6, we show the number-average cluster size, calculated from the CSD according to equation (8) with $i=5$, as well as the mass-average cluster size $\left(C S_{\mathrm{M}}\right)$, computed via:

$$
\left\langle C S_{\mathrm{M}}\right\rangle_{5}=\frac{\sum_{n=5}^{\infty} n^{2}\left[\mathrm{M}_{n}\right]}{\sum_{n=5}^{\infty} n\left[\mathrm{M}_{n}\right]}
$$

A few experimental studies ${ }^{31,33-36}$ present values for the $C S_{\mathrm{M}}$ of DeTAB at room temperature ranging from 31 to 40 . These values are all somewhat higher than our simulation results at $353 \mathrm{~K}$. However, the average aggregation number decreases with temperature ${ }^{36}$, so this seems consistent with our simulation results. Additionally, one may also obtain a rough estimate of the critical micelle concentration from the amount of free surfactant molecules that remain in solution after the aggregation process. To be consistent with our calculations of the average cluster size, we estimate the cmc from the concentration of surfactants in aggregates with less than 5 molecules (as aggregates with 5 molecules or more are considered as micelles). These results are also shown in Table 6. The experimental value of the $\mathrm{cmc}$ at $353 \mathrm{~K}$ may be estimated by interpolating between results obtained at several temperatures ${ }^{35}$. This procedure yields an experimental cmc of $0.085 \mathrm{~mol} / \mathrm{l}$, which lies somewhat above our simulation values. Our systems are clearly too small to allow for an accurate determination of the $\mathrm{cmc}^{11}$, at least without employing grand canonical ensemble simulations. Nevertheless, it is quite encouraging that they are not very far off, particularly for the UA model.

\section{Table 6}

As we have described previously, our analysis procedure allows us to identify individual micelles in each sampled configuration. Once this is done, one may extract several important structural properties of each aggregate. In Figure 9 we show a typical snapshot of an individual micelle for each of the surfactant models. Qualitatively speaking, both micelles look very similar 
- the tail atoms cluster near the center, the head atoms are near the micelle surface and the bromide counterions are somewhat loosely dispersed around the head region. A more quantitative analysis of the micellar structure is provided by the density profiles measured in spherical shells radiating from the micelle COM. These profiles are shown in Figure 10 for micelles of two different sizes. In both cases, and for both surfactant models, we can clearly identify a core region composed of only tail atoms, which create a very hydrophobic environment that completely excludes water molecules. As we move toward the micelle surface, we begin to observe a rise in the water density and a peak corresponding to the surfactant head groups. The appearance of this peak marks a transition between the hydrophobic micelle core and the hydrophilic micelle shell. One can also observe the presence of a Stern layer of bound counterions, located on the exterior of the micelle about $0.3 \mathrm{~nm}$ away from the head-group peak. Strikingly, the density profiles for the UA and the AA models are almost identical, the main difference being a slightly higher density in the head-group layer for the latter.

\section{Figure 9}

Figure 10

It is also possible to calculate average measures of the micelle size and shape. The micelle radius $\left(R_{M}\right)$, may be estimated by the location of the maximum in the average head-group density profile, relative to the micelle COM. These values are shown in Table 6 for both models. Using the value of $R_{M}$ and the average aggregation number, we can compute the average surface area per head group (a). These results may be compared to available experimental values at 298 $\mathrm{K}^{35,37,38}$, which are between 0.63 and $0.80 \mathrm{~nm}^{2}$. Our simulation results are somewhat above the experimental values, but the discrepancy might be simply due to the different temperatures - at higher temperature, the aggregation number decreases and the micelles become more disordered, leading to an increase in $a$. 
We have also calculated the moments of inertia tensor and, from it, the radius of gyration of each micelle. The first step is to translate the coordinates of all surfactant molecules that belong to the micelle so that the micelle COM is at the origin. The moments of inertia tensor (I) for a given micelle is then calculated from:

$$
\mathbf{I}=\left[\begin{array}{ccc}
\frac{\sum_{i} m_{i}\left(y_{i}^{2}+z_{i}^{2}\right)}{2 \sum_{i} m_{i}} & \frac{-\sum_{i} m_{i} x_{i} y_{i}}{2 \sum_{i} m_{i}} & \frac{-\sum_{i} m_{i} x_{i} z_{i}}{2 \sum_{i} m_{i}} \\
\frac{-\sum_{i} m_{i} x_{i} y_{i}}{2 \sum_{i} m_{i}} & \frac{\sum_{i} m_{i}\left(x_{i}^{2}+z_{i}^{2}\right)}{2 \sum_{i} m_{i}} & \frac{-\sum_{i} m_{i} y_{i} z_{i}}{2 \sum_{i} m_{i}} \\
\frac{-\sum_{i} m_{i} x_{i} z_{i}}{2 \sum_{i} m_{i}} & \frac{-\sum_{i} m_{i} y_{i} z_{i}}{2 \sum_{i} m_{i}} & \frac{\sum_{i} m_{i}\left(x_{i}^{2}+y_{i}^{2}\right)}{2 \sum_{i} m_{i}}
\end{array}\right]
$$

where $x_{i}, y_{i}$ and $z_{i}$ are the coordinates of atom $i$ of a surfactant molecule belonging to the micelle and $m_{i}$ is its mass. Notice the normalization term in the denominator of each element of the matrix. The tensor is then diagonalized, by calculating its eigenvectors, to obtain the principal moments of inertia ( $I_{x x}, I_{y y}$ and $I_{z z}$, ordered from the smallest to the largest). These provide information on the shape of the micelle, and allow for the calculation of its gyration radius $\left(R_{g}\right)$ :

$$
R_{g}=\sqrt{I_{x x}+I_{y y}+I_{z z}}
$$

The results of this procedure are also shown in Table 6 . We can see that the micelles are almost spherical, with a slight tendency for a prolate ellipsoid shape (in which $I_{x x}<I_{y y}=I_{z z}$ ).

A more detailed picture of the interactions at the micelle surface may be obtained by analyzing radial distribution functions (RDFs) between different atoms. Figure 11 shows the RDFs between surfactant nitrogen atoms and bromide ions. Both models show one large, somewhat broad peak around $0.5 \mathrm{~nm}$, corresponding to the counterion Stern layer located on the exterior of the micelle. This layer extends up to a distance of about $0.65 \mathrm{~nm}$ from the position of 
the nitrogen atoms. The minimum is quite shallow and it is difficult to pinpoint its location with precision. The RDFs show that the bromide ions are more strongly attracted to the AA head groups than to the UA head groups, since the latter shows a shorter and broader peak. These differences in local binding between counterions and surfactant heads are probably due to the consideration of explicit hydrogens in the AA model, which allow for a more efficient packing of bromide ions around the head groups. However, these differences in local structure seem to have a small effect on the self-assembly process and on global properties of the system (such as the average micelle size).

Integration of the $\mathrm{RDF}$ up to the first minimum can provide an estimate of the degree of counterion dissociation $(\beta)$. This is basically 1 minus the number of bound bromide ions per head group on the micelle surface, and is given by:

$$
\beta=1-4 \pi \rho_{\mathrm{Br}} \int_{0}^{r_{\min }} g_{\mathrm{N}-\mathrm{Br}}(r) r^{2} \mathrm{~d} r
$$

where $g(r)$ is the RDF, $r_{\min }$ is its first minimum (in this case, it is located at $0.65 \mathrm{~nm}$ ), and $\rho_{\mathrm{Br}}$ is the number density of bromide ions in the simulation cell (i.e., the total number of $\mathrm{Br}$ ions divided by the average cell volume). $\beta$ was also calculated directly from the MD trajectories, by counting the number of $\mathrm{Br}$ ions that were located less than $0.65 \mathrm{~nm}$ away from each nitrogen atom. The results were nearly the same as those obtained from the RDFs, and are shown in Table 6. Experimental values for $\beta$ at $298 \mathrm{~K}^{32,35,38-41}$ are between 0.22 and 0.30 . The simulation result for the UA model is within this range of values, while the AA model underestimates the degree of dissociation. However, it is expected that $\beta$ will increase with temperature, given that the aggregation number decreases and the micelles will become more disordered due to thermal fluctuations, so it seems that both models are overestimating the strength of the interaction between bromide ions and surfactant head groups. Nevertheless, the simulation results are 
somewhat sensitive to the distance threshold below which a counterion is said to be bound to the micelle. For instance, reducing this threshold to $0.625 \mathrm{~nm}$, which is still acceptable based on the N-Br RDFs, changes the values of $\beta$ to 0.19 for the AA model and to 0.35 for the UA model. This variation is due to the rather diffuse nature of the Stern layer, which prevents a precise determination of the minimum in the RDF and, hence, hinders the quantification of the degree of counterion dissociation.

\section{Figure 11}

Interesting information can also be gleaned from the RDFs that describe interactions between water molecules and surfactants, shown in Figure 12. The distribution between water oxygens (Ow) and $\mathrm{MH}$ groups shows a first sharp peak at $0.36 \mathrm{~nm}$ followed by a second more diffuse peak at $0.54 \mathrm{~nm}$. The first peak is due to strong electrostatic interactions between an Ow atom (with a negative partial charge) and one of the MH groups (with positive partial charges, see Table 1). The second peak is due to the other two MH groups, not interacting directly with that water oxygen. Interestingly, the RDF between $\mathrm{Ow}$ atoms and the $\mathrm{EH}$ group (linking the nitrogen to the aliphatic chain - see Figure 1) shows two peaks at the same positions as the Ow-MH RDF. This clearly indicates that some water molecules are able to migrate into the head-group layer and establish a close interaction with the EH group. The strong hydrophobic environment beyond the head-group layer, however, means that only a few water molecules are allowed to interact with $\mathrm{EH}$ atoms, and thus the height of the first peak is significantly reduced relative to that of $\mathrm{Ow}-\mathrm{MH}$ interactions. The RDF between Ow and the first ET group of the aliphatic chain shows no evidence of close interactions - the peaks at $0.46 \mathrm{~nm}$ and $0.65 \mathrm{~nm}$ are merely a reflection of the Ow-EH interactions.

\section{Figure 12}


The picture emerging from our results shows that bromide counterions are mainly located on the outside of the micelle surface, in close contact with the surfactant head groups. Water molecules hydrate the counterions and the surfactant head, and a few are able to migrate past the head-group layer, interacting with inner head-group carbons. This structure is illustrated in Figure 13, which depicts a snapshot of a cross-section of the simulation box, blown up to focus on a section of a typical micelle. The picture observed in Figure 13 and deduced from our results is in good agreement with a previous MD study of a single DeTAB micelle ${ }^{5}$ and with a recent experimental description based on dielectric spectroscopic studies of alkyltrimethylammonium bromide micellar systems ${ }^{42}$.

\section{Figure 13}

\section{Conclusions}

In this paper, we have presented a detailed look at the self-assembly of DeTAB micelles using MD simulations. The simulated systems are large enough to allow the formation of around 10 micellar aggregates, and are long enough to permit an approach to thermodynamic equilibrium. The self-assembly proceeds in several stages. In the first few picoseconds, surfactant monomers quickly aggregate to form oligomers and a few small micelles. The oligomers are quite disordered and unstable, so they gradually dissolve providing material for the growth of more stable, larger aggregates. This "ripening" process lasts until about 700 ps of simulation time. Once this is concluded, the system is mainly composed of free monomers and dimers, together with a distribution of individual micelles. Further increases in the average aggregate size take place by occasional collisions between individual micelles.

The first two stages of self-assembly are dominated by aggregation of monomers and small oligomers. As such, the first 700 ps are well described by a simplified kinetic model based on diffusion-limited surfactant aggregation with a size-independent rate constant estimated from 
the diffusion coefficient and collision radius of an isolated $\mathrm{DeTA}^{+}$cation. Beyond $700 \mathrm{ps}$, the model is no longer able to describe the kinetics of the process, which could be due to several factors. Firstly, the model entirely neglects the dissociation process, whereas dissociation is necessarily important close to equilibrium. The neglect of cluster dissociation means that the model predicts a zero concentration of monomers and infinite-sized clusters at infinite time (see equations (6) and (9)), which is clearly unrealistic. Secondly, in later stages of the process, the system evolves mostly by collisions between relatively large micelles, and thus a rate constant based on the diffusion of isolated monomers is no longer adequate. Finally, it is also likely that potential interactions between clusters, which are not considered in the model, play an important part in this later stage.

After about $7 \mathrm{~ns}$, collision events become less frequent, and the system approaches equilibrium. We cannot, of course, be certain that our simulations have reached full thermodynamic equilibrium. However, some results suggest that they may not be very far from equilibrium. First of all, the properties of the system (such as the average cluster size, the concentration of free surfactants and the micellar radius of gyration) fluctuate around fixed values during a long period of time (about $7 \mathrm{~ns}$ ). The average CSD during this time period shows a bimodal shape, with a peak for monomers and small oligomers clearly separated from a region of large micelles, which is what is expected for a micellar solution at equilibrium. Finally, we are able to observe several processes that are typical of a system approaching equilibrium, such as incorporation and detachment of surfactant molecules from individual micelles, complete micellar dissolution, and fusion between two micelles. In fact, to our knowledge it is the first time that an event of micelle fusion has been observed and described in a simulation using realistic atomistic models. 
In a real surfactant solution, occasional fusion events will lead to the formation of large micelles that are well above the equilibrium average size. These micelles will be unstable, and will eventually dissolve or break up into smaller aggregates. During the course of our simulations, we have observed complete dissolution of a small micelle, which took place by gradual loss of monomers and dimers, but have not observed any event during which a large micelle broke up into two smaller aggregates. However, one cannot rule out the possibility that larger micelles will break up into two smaller, but stable clusters, in a process that is essentially the reverse of the fusion event described in section 3.1. To examine whether this is indeed the case, and to further assess if our simulations correspond to an equilibrated state, one could start from a configuration with an average micelle size that is well above the expected value at equilibrium (say, a single micelle composed of 125 molecules, or two micelles with 62 surfactants each). In their simulations of self-assembly, Maillet et al. ${ }^{13}$ have started from preformed large micelles and have observed their breakup into two smaller aggregates. However, the intrinsic stability of a large micelle that is not formed by spontaneous aggregation is questionable. A more realistic option would be to extend our runs until one such large micelle is spontaneously formed, and then monitor its evolution in time. Such a simulation, however, would be extremely time-consuming.

Using a cluster-counting algorithm, we were able to identify individual micelles and calculate important structural properties of the micellar system. The simulations provide reasonable estimates of the average cluster size, the area per head-group, the degree of counterion dissociation and the critical micelle concentration. Nevertheless, more accurate methods to calculate these properties from simulation data would be useful (particularly in the case of $\mathrm{cmc}$ and $\beta$ ). On average, the DeTAB micelles are almost spherical, with a slight tendency for a prolate ellipsoidal shape. The core of the micelles is composed entirely of tail atoms and completely 
excludes water molecules. Surrounding the core is a layer composed mostly of surfactant head atoms solvated by water molecules. These preferentially interact with the positively charged $\mathrm{MH}$ groups located on the exterior of the micelle, but a few of them are able to penetrate the headgroup layer and interact with the inner EH groups. Most of the bromide counterions are closely associated with the cationic head groups, forming a Stern layer that is located just outside of the micelle surface.

Finally, we have compared the behavior of two realistic surfactant models: one based on a united-atom approach, and the other based on an all-atom description, with explicit hydrogens. Generally speaking, the self-assembly process and the system properties at equilibrium are not strongly affected by the choice of model. The AA model provides a slightly better estimate of the $\operatorname{DeTA}^{+}$diffusion coefficient, while the UA model yields values of the average micelle size and structure that are closer to experimental results. Since the UA model is more computationally efficient (by a factor of about 1.4 for the systems studied), we recommend it for use in subsequent simulation studies involving alkylammonium surfactants. We have already applied this model in a study of the early stages of surfactant-templated synthesis of periodic mesoporous silicas ${ }^{43}$, and we intend to pursue this line of research in the near future.

\section{Acknowledgments:}

The author gratefully acknowledges Nigel A. Seaton for fruitful discussions and helpful suggestions. Partial funding for this work was provided by the HPC-Europa programme, funded under the European Commission's Research Infrastructures activity of the Stucturing the European Research Area programme, contract number RII3-CT-2003-506079.

\section{References:}

[1] Israelachvili, J. N. Intermolecular and Surface Forces; Academic Press: London, UK, 1985. 
[2] Gelbart, W. M.; Ben-Shaul, A. J. Phys. Chem. 1996, 100, 13169.

[3] Shelley, J. C.; Shelley, M. Y. Curr. Opin. Colloid Interface Sci. 2000, 5, 101.

[4] Böcker, J; Brickmann, J.; Bopp, P. J. Phys. Chem. 1994, 98, 712.

[5] Pal, S.; Bagchi, B.; Balasubramanian, S. J. Phys. Chem. 2005, 109, 12879.

[6] Jury, S; Bladon, P.; Cates, M; Krishna, S.; Hagen, M.; Ruddock, N.; Warren, P. Phys. Chem. Chem. Phys. 1999, 1, 2051.

[7] Boghosian, B. M.; Coveney, P. V.; Love, P. J. Proc. R. Soc. Lond. A 2000, 456, 1431.

[8] Smit, B.; Esselink, K.; Hilbers, P. A. J.; van Os, N. M.; Rupert, L. A. M.; Szleifer, I. Langmuir 1993, 9, 9.

[9] Palmer, B. J.; Liu, J. Langmuir 1996, 12, 746.

[10] Larson, R. G.; Scriven, L. E.; Davis, H. T. J. Chem. Phys. 1985, 83, 2411.

[11] Mackie, A. D.; Panagiotopoulos, A. Z.; Szleifer, I. Langmuir 1997, 13, 5022.

[12] Tarek, M.; Bandyopadhyay, S.; Klein, M. L. J. Mol. Liq. 1998, 78, 1.

[13] Maillet, J.-B.; Lachet, V.; Coveney, P. V. Phys. Chem. Chem. Phys. 1999, 1, 5277.

[14] Salaniwal, S.; Cui, S. T.; Cummings, P. T.; Cochran, H. D. Langmuir 1999, 15, 5188.

[15] Marrink, S. J.; Tieleman, D. P.; Mark, A. E. J. Phys. Chem. B 2000, 104, 12165.

[16] Berendsen, H. J. C.; van der Spoel, D.; van Drunen, R. Comp. Phys. Comm. 1995, 91, 43.

[17] Lindahl, E.; Hess, B.; van der Spoel, D. J. Mol. Mod. 2001, 7, 306. 
[18] Hockney, R. W.; Goel, S. P. J. J. Comput. Phys. 1974, 14, 148.

[19] Nosé, S. Mol. Phys. 1984, 52, 255.

[20] Hoover, W. G. Phys. Rev. A 1985, 31, 1695.

[21] Parrinello, M.; Rahman, A. J. Appl. Phys. 1981, 52, 7182.

[22] Berendsen, H. J. C.; Grigera, J. R.; Straatsma, T. P. J. Phys. Chem. 1997, 91, 6269.

[23] Jorgensen, W. L.; Maxwell, D. S.; Tirado-Rives, J. J. Am. Chem. Soc. 1996, 118, 11225.

[24] Jorgensen, W. L.; Gao, J. J. Phys. Chem. 1986, 90, 2174.

[25] Smit, B.; Karaborni, S.; Siepmann, J. I. J. Chem. Phys. 1995, 102, 2126.

[26] Hess, B.; Bekker, H.; Berendsen, H. J. C.; Fraaije, J. G. E. M. J. Comp. Chem. 1997, 18, 1463.

[27] Essman, U.; Perela, L.; Berkowitz, M. L.; Darden, T.; Lee, H.; Pedersen, L. G. J. Chem. Phys. 1995, 103, 8577.

[28] Hoshen, J.; Kopelman, R. Phys. Rev. B 1976, 14, 3438.

[29] Ratke, L.; Voorhees, P. W. (Eds) Growth and Coarsening: Ostwald Ripening in Material Processing; Springer-Verlag Telos: New York, 2002.

[30] Haile, J. M. Molecular Dynamics Simulation; Wiley: New York, 1997.

[31] D’Errico, G.; Ortona, O.; Paduano, L.; Vitagliano, V. J. Colloid Interface Sci. 2001, 239, 264. 
[32] Ribeiro, A. C. F.; Lobo, V. M. M.; Valente, A. J. M.; Azevedo, E. F. G.; Miguel M. G.; Burrows, H. D. Colloid Polym. Sci. 2004, 283, 277.

[33] Yoshida, N.; Matsuoka, K.; Moroi, Y. J. Colloid Interface Sci. 1997, 187, 388.

[34] Nomura, H.; Koda, S.; Matsuoka, T.; Hiyama, T.; Shibata, R.; Kato, S. J. Colloid Interface Sci. 2000, 230, 22.

[35] Evans, D. F.; Allen, M.; Ninham, B. W.; Fouda, A. J. Sol. Chem. 1984, 13, 87.

[36] Dorrance, R. C.; Hunter, T. F. J. Chem. Soc., Faraday Trans. 1 1974, 70, 1572.

[37] Al-Wardian, A.; Glenn, K. M.; Palepu, R. M. Colloids Surf. A: Physicochem. Eng. Aspects 2004, 247, 115 .

[38] Zana, R. J. Colloid Interface Sci. 1980, 78, 330.

[39] de Lisi, R.; Gradzielski, M.; Lazzara, G.; Milioto, S.; Muratore, N.; Prevost, S. J. Phys. Chem. B 2006, 110, 25883.

[40] del Rio, J. M.; Pombo, C.; Prieto, G.; Mosquera, V.; Sarmiento, F. J. Colloid Interface Sci. 1995, 172, 137.

[41] Mosquera, V.; del Rio, J. M.; Attwood, D.; Garcia, M.; Jones, M. N.; Prieto, G.; Suarez, M. J.; Sarmiento, F. J. Colloid Interface Sci. 1998, 206, 66.

[42] Buchner, R.; Baar, C.; Fernandez, P.; Schrödle, S.; Kunz, W. J. Mol. Liq. 2005, 118, 179.

[43] Jorge, M.; Gomes, J. R. B.; Cordeiro, M. N. D. S.; Seaton, N. A. J. Am. Chem. Soc. 2007, $129,15414$. 
Table 1 - Intermolecular parameters for both surfactant models.

\begin{tabular}{|c|c|c|c|c|c|c|c|c|}
\hline & \multicolumn{4}{|c|}{ All-Atom } & \multicolumn{3}{c|}{ United-Atom } \\
\hline Atom & Mass (a.u.) & $\sigma(\mathrm{nm})$ & $\varepsilon\left(\mathrm{kJ} \mathrm{mol}^{-1}\right)$ & q (a.u.) & Mass (a.u.) & $\sigma(\mathrm{nm})$ & $\varepsilon\left(\mathrm{kJ} \mathrm{mol}^{-1}\right)$ & q (a.u.) \\
\hline $\mathrm{N}$ & 14.007 & 0.325 & 0.7113 & 0.00 & 14.007 & 0.325 & 0.7113 & 0.00 \\
\hline $\mathrm{MH}$ & 12.011 & 0.350 & 0.2761 & 0.07 & 15.035 & 0.396 & 0.6067 & 0.25 \\
\hline $\mathrm{EH}$ & 12.011 & 0.350 & 0.2761 & 0.13 & 14.027 & 0.396 & 0.6067 & 0.25 \\
\hline $\mathrm{MT}$ & 12.011 & 0.350 & 0.2761 & -0.18 & 15.035 & 0.393 & 0.9478 & 0.00 \\
\hline $\mathrm{ET}$ & 12.011 & 0.350 & 0.2761 & -0.12 & 14.027 & 0.393 & 0.3908 & 0.00 \\
\hline $\mathrm{H}$ & 1.008 & 0.250 & 0.1255 & 0.06 & -- & -- & - & - \\
\hline $\mathrm{Br}$ & 79.904 & 0.462 & 0.3766 & -1.00 & 79.904 & 0.462 & 0.3766 & -1.00 \\
\hline
\end{tabular}


Table 2 - Bond lengths (in $\mathrm{nm}$ ) for both surfactant models.

\begin{tabular}{|c|c|c|}
\hline Bond & All-Atom & United-Atom \\
\hline N-C & 0.1471 & 0.1471 \\
\hline C-C & 0.1529 & 0.1540 \\
\hline C-H & 0.1090 & -- \\
\hline
\end{tabular}


Table 3 - Harmonic angle potential parameters for both surfactant models.

\begin{tabular}{|c|c|c|c|c|}
\hline & \multicolumn{2}{|c|}{ All-Atom } & \multicolumn{2}{c|}{ United-Atom } \\
\hline Angle & $\theta_{0}(\mathrm{deg})$ & $k_{\theta}\left(\mathrm{kJ} \mathrm{mol}^{-1} \mathrm{rad}^{-2}\right)$ & $\theta_{0}(\mathrm{deg})$ & $k_{\theta}\left(\mathrm{kJ} \mathrm{mol}^{-1} \mathrm{rad}^{-2}\right)$ \\
\hline $\mathrm{C}-\mathrm{N}-\mathrm{C}$ & 113.0 & 418.40 & 113.0 & 418.40 \\
\hline $\mathrm{N}-\mathrm{C}-\mathrm{C}$ & 111.2 & 669.44 & 111.2 & 669.44 \\
\hline $\mathrm{N}-\mathrm{C}-\mathrm{H}$ & 109.5 & 292.88 & -- & -- \\
\hline $\mathrm{C}-\mathrm{C}-\mathrm{C}$ & 112.7 & 488.27 & 114.0 & 519.63 \\
\hline $\mathrm{C}-\mathrm{C}-\mathrm{H}$ & 110.7 & 313.80 & -- & -- \\
\hline $\mathrm{H}-\mathrm{C}-\mathrm{H}$ & 107.8 & 276.14 & -- & - \\
\hline
\end{tabular}


Table 4 - Ryckaert-Bellemans torsional potential parameters (in $\mathrm{kJ} \mathrm{mol}^{-1}$ ) for both surfactant models.

\begin{tabular}{|c|c|c|c|c|c|c|c|c|}
\hline & \multicolumn{5}{|c|}{ All-Atom } & \multicolumn{4}{c|}{ United-Atom } \\
\hline Dihedral & $C_{0}$ & $C_{1}$ & $C_{2}$ & $C_{3}$ & $C_{0}$ & $C_{1}$ & $C_{2}$ & $C_{3}$ \\
\hline N-C-C-C & 5.772 & -2.671 & 0.958 & -4.058 & 8.397 & 16.786 & 1.134 & -26.317 \\
\hline C-N-C-C & 3.042 & -1.351 & 0.519 & -2.209 & 3.042 & -1.351 & 0.519 & -2.209 \\
\hline N-C-C-H & 0.803 & 2.410 & 0.000 & -3.213 & -- & -- & -- & -- \\
\hline C-N-C-H & 0.632 & 1.895 & 0.000 & -2.527 & -- & -- & -- & -- \\
\hline C-C-C-C & 2.929 & -1.464 & 0.209 & -1.674 & 8.397 & 16.786 & 1.134 & -26.317 \\
\hline C-C-C-H & 0.628 & 1.883 & 0.000 & -2.510 & -- & -- & -- & - \\
\hline H-C-C-H & 0.628 & 1.883 & 0.000 & -2.510 & -- & -- & -- & -- \\
\hline
\end{tabular}


Table 5 - Parameters used in the theoretical kinetic aggregation model.

\begin{tabular}{|c|c|c|}
\hline & All-Atom & United-Atom \\
\hline \multirow{2}{*}{$D \times 10^{9}\left(\mathrm{~m}^{2} / \mathrm{s}\right)$} & 1.872 & 1.912 \\
\cline { 2 - 3 } & $0.744^{\mathrm{a}}$ & $0.770^{\mathrm{a}}$ \\
\hline$R(\mathrm{~nm})$ & 0.549 & 0.532 \\
\hline$L(\mathrm{~nm})$ & 6.304 & 6.292 \\
\hline$\tau(\mathrm{ps})$ & 77.57 & 77.98 \\
\hline
\end{tabular}

${ }^{\text {a }}$ Calculated at $T=298.15 \mathrm{~K}$. 
Table 6 - Properties of the micellar systems averaged over the last 7 ns of each simulation run.

\begin{tabular}{|c|c|c|}
\hline & All-Atom & United-Atom \\
\hline$\left\langle C S_{\mathrm{N}}\right\rangle$ & 20.32 & 22.28 \\
\hline$\left\langle C S_{\mathrm{M}}\right\rangle$ & 22.05 & 24.89 \\
\hline $\mathrm{cmc}(\mathrm{mol} / \mathrm{l})$ & 0.027 & 0.051 \\
\hline$R_{M}(\mathrm{~nm})$ & 1.230 & 1.311 \\
\hline$a\left(\mathrm{~nm}^{2}\right)$ & 0.862 & 0.868 \\
\hline$I_{x x}\left(\mathrm{~nm}^{2}\right)$ & 0.356 & 0.372 \\
\hline$I_{y y}\left(\mathrm{~nm}^{2}\right)$ & 0.465 & 0.487 \\
\hline$I_{z z}\left(\mathrm{~nm}^{2}\right)$ & 0.522 & 0.545 \\
\hline$R_{g}\left(\mathrm{~nm}^{2}\right.$ & 1.159 & 1.185 \\
\hline$\beta$ & 0.103 & 0.218 \\
\hline
\end{tabular}


Figure 1. Diagram of a $\mathrm{DeTA}^{+}$cation showing the nomenclature for atom types: $\mathrm{N}$ corresponds to a nitrogen atom, $\mathrm{MH}$ is a methyl group belonging to the surfactant head, $\mathrm{EH}$ is a head methylene group, MT is a tail methyl group and ET is a tail methylene group. The all-atom model considers explicit hydrogen atoms (two in each methylene group and three in each methyl group), but these are not shown for clarity of representation.

Figure 2. Evolution in time of the self-assembly simulations using the united-atom (thick lines) and the all-atom (thin lines) models. Panel a) shows the mass-average cluster size (considering only clusters with 5 or more molecules) and panel b) shows the total number of clusters in the simulation box.

Figure 3. Evolution of the cluster size distribution for the simulation with the united-atom model. Panel a) was obtained between 60 and 100 ps of simulation time, panel b) between 260 and 300 ps, panel c) between 460 and 500 ps and panel d) between 660 and 700 ps.

Figure 4. Snapshots of typical oligomers formed during the early stages of DeTAB selfassembly: (a) pentamer from the all-atom run; (b) trimer from the united-atom run. Surfactant tail atoms are represented by green spheres, head atoms by purple spheres, hydrogen atoms by white spheres and bromide ions by grey spheres. Water molecules have been omitted for clarity.

Figure 5. Comparison between simulation results (thin lines) and kinetic theory predictions (smooth thick lines) for the time evolution of the surfactant aggregation process, using: a) the united-atom model; b) the all-atom model.

Figure 6. Snapshots of the united-atom simulation showing the fusion of two micelles. Simulation times are: a) 2180 ps; b) 4260 ps; c) 4300 ps; d) 4360 ps; e) 4600 ps. Color coding is the same as in Figure 4. Water molecules have been omitted for clarity. 
Figure 7. Plot showing the fusion of two micelles, corresponding to the snapshots of Figure 6. Panel a) shows the evolution of the size of both micelles, while panel b) plots the $x, y$ and $z$ coordinates of the center-of-mass of both micelles. Thin lines are for the smaller micelle (on the right in Figure 6) and thick lines are for the larger micelle (on the left in Figure 6). The fusion takes place at $4360 \mathrm{ps}$.

Figure 8. Cluster size distributions for the united-atom (thick line) and all-atom (thin line) models, averaged over the last $7 \mathrm{~ns}$ of simulation time.

Figure 9. Snapshots of typical micelles obtained during the simulation runs with the united-atom (a) and all-atom (b) models. Color coding is the same as in Figure 4 and water molecules have been omitted for clarity.

Figure 10. Comparison between the density profiles of micelles obtained using the united-atom (thick lines) and all-atom (thin lines) models. Panel a) shows the profiles for micelles containing 15 or 16 surfactant molecules, while panel b) is for micelles with 29 or 30 surfactants.

Figure 11. Radial distribution functions between surfactant nitrogen atoms and bromide counterions for the united-atom (thick line) and all-atom (thin line) models.

Figure 12. Radial distribution functions, for the all-atom model, between water oxygen atoms and carbon atoms in the surfactant molecule: $\mathrm{MH}$ outer head carbons (thick line); EH inner head carbon (dashed line); ET1 first tail carbon (thin line).

Figure 13. Snapshot of a cross-section of the simulation box in the AA run. The snapshot is blown up to show the structure of a typical micelle in detail. Color coding is the same as in Figure 4, except for water oxygens, which are represented by blue spheres. 


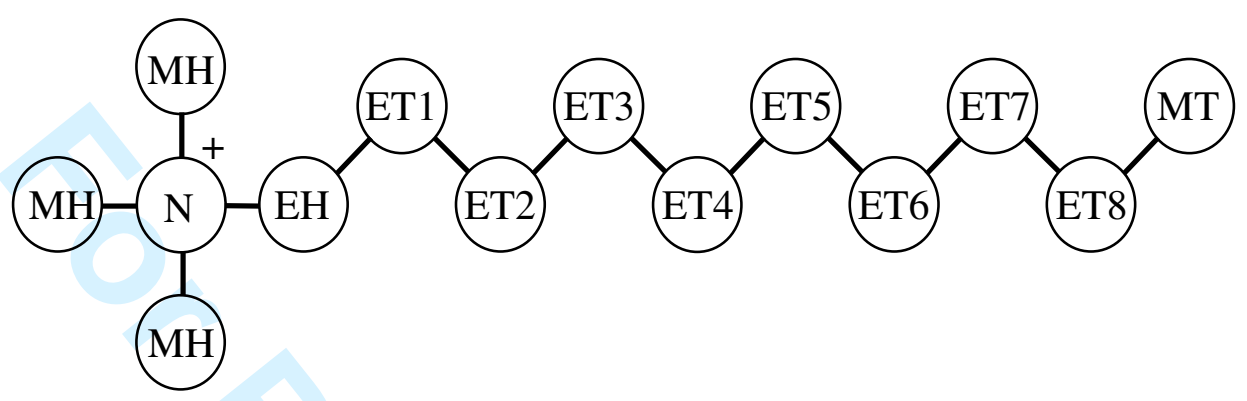

Figure 1. Diagram of a DeTA ${ }^{+}$cation showing the nomenclature for atom types: $\mathrm{N}$ corresponds to a nitrogen atom, MH is a methyl group belonging to the surfactant head, EH is a head methylene group, MT is a tail methyl group and ET is a tail methylene group. The all-atom model considers explicit hydrogen atoms (two in each methylene group and three in each methyl group), but these are not shown for clarity of representation. 

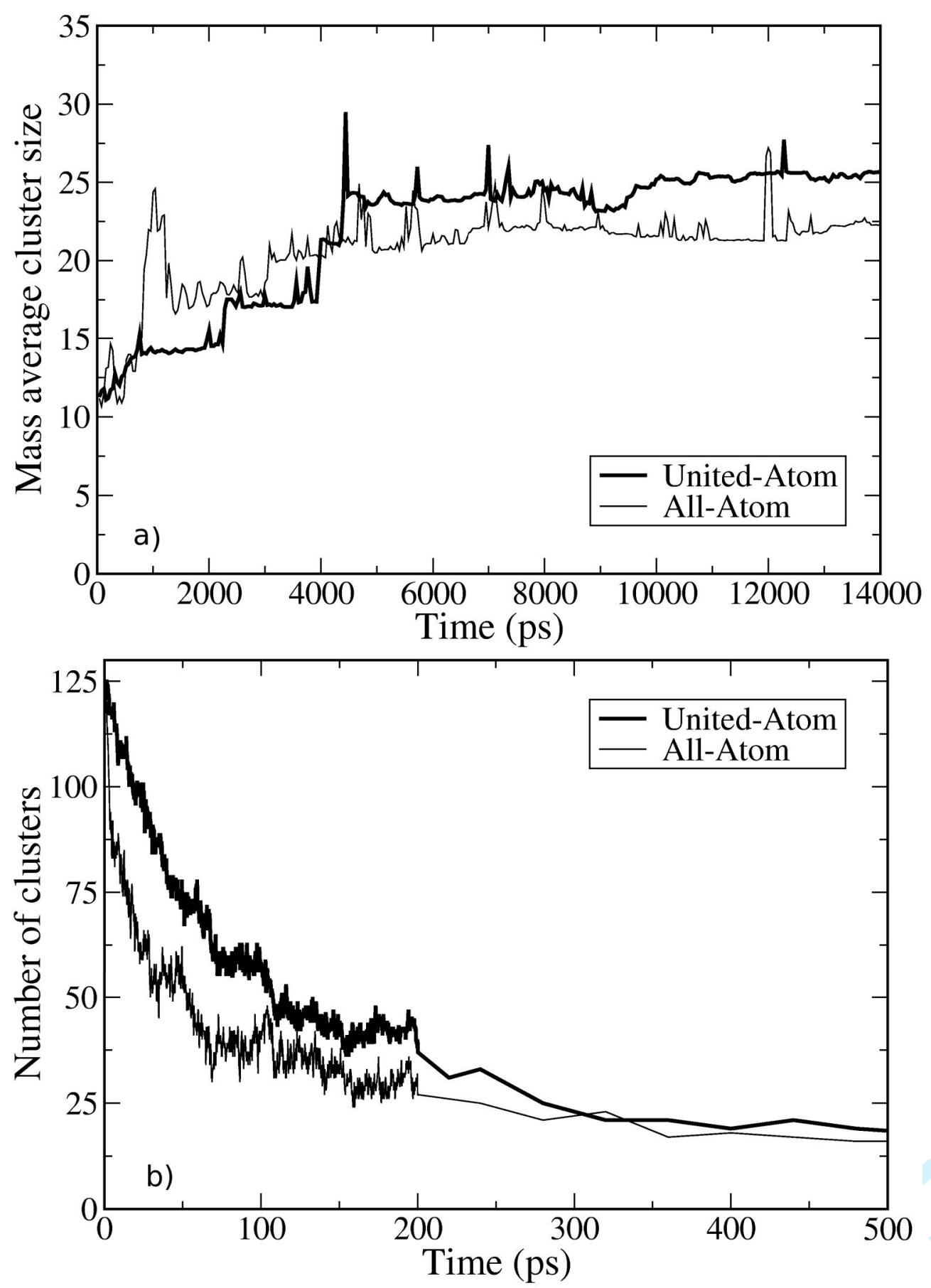

Figure 2. Evolution in time of the self-assembly simulations using the united-atom (thick lines) and the all-atom (thin lines) models. Panel a) shows the mass-average cluster size (considering only clusters with 5 or more molecules) and panel b) shows the total number of clusters in the simulation box. 

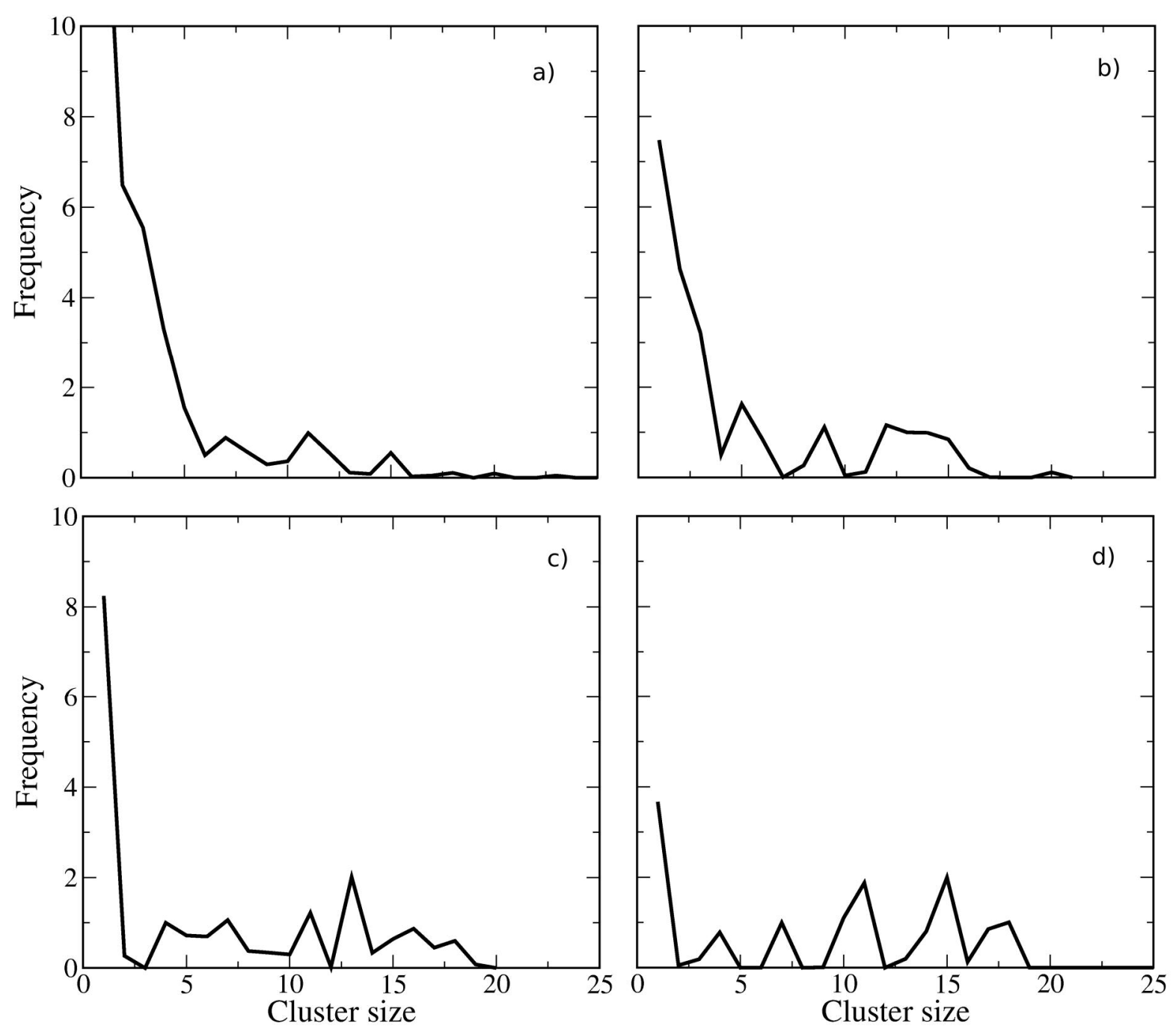

Figure 3. Evolution of the cluster size distribution for the simulation with the united-atom model.

Panel a) was obtained between 60 and 100 ps of simulation time, panel b) between 260 and 300 ps, panel c) between 460 and 500 ps and panel d) between 660 and 700 ps. 

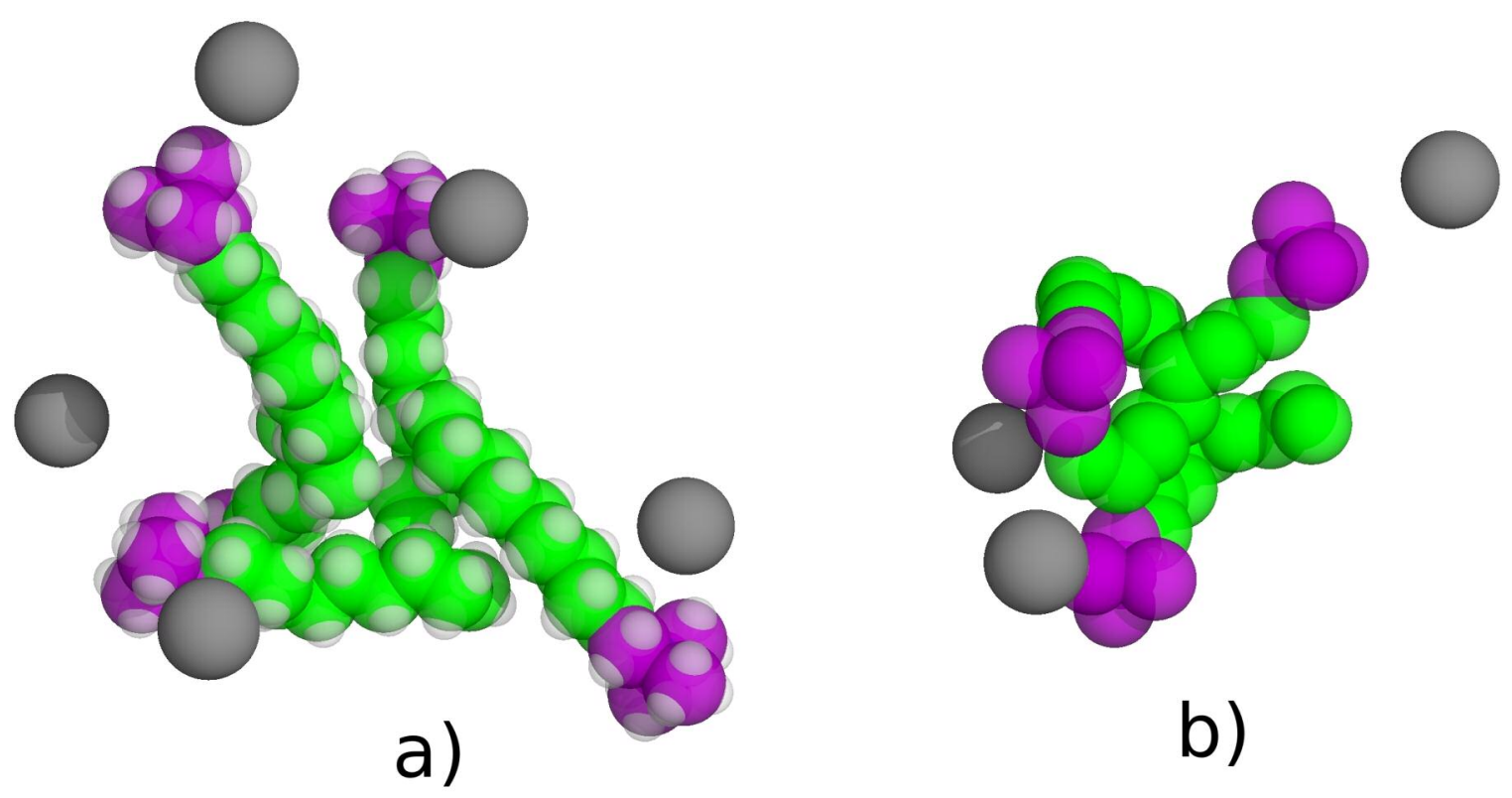

b)

Figure 4. Snapshots of typical oligomers formed during the early stages of DeTAB selfassembly: (a) pentamer from the all-atom run; (b) trimer from the united-atom run. Surfactant tail atoms are represented by green spheres, head atoms by purple spheres, hydrogen atoms by white spheres and bromide ions by grey spheres. Water molecules have been omitted for clarity. 

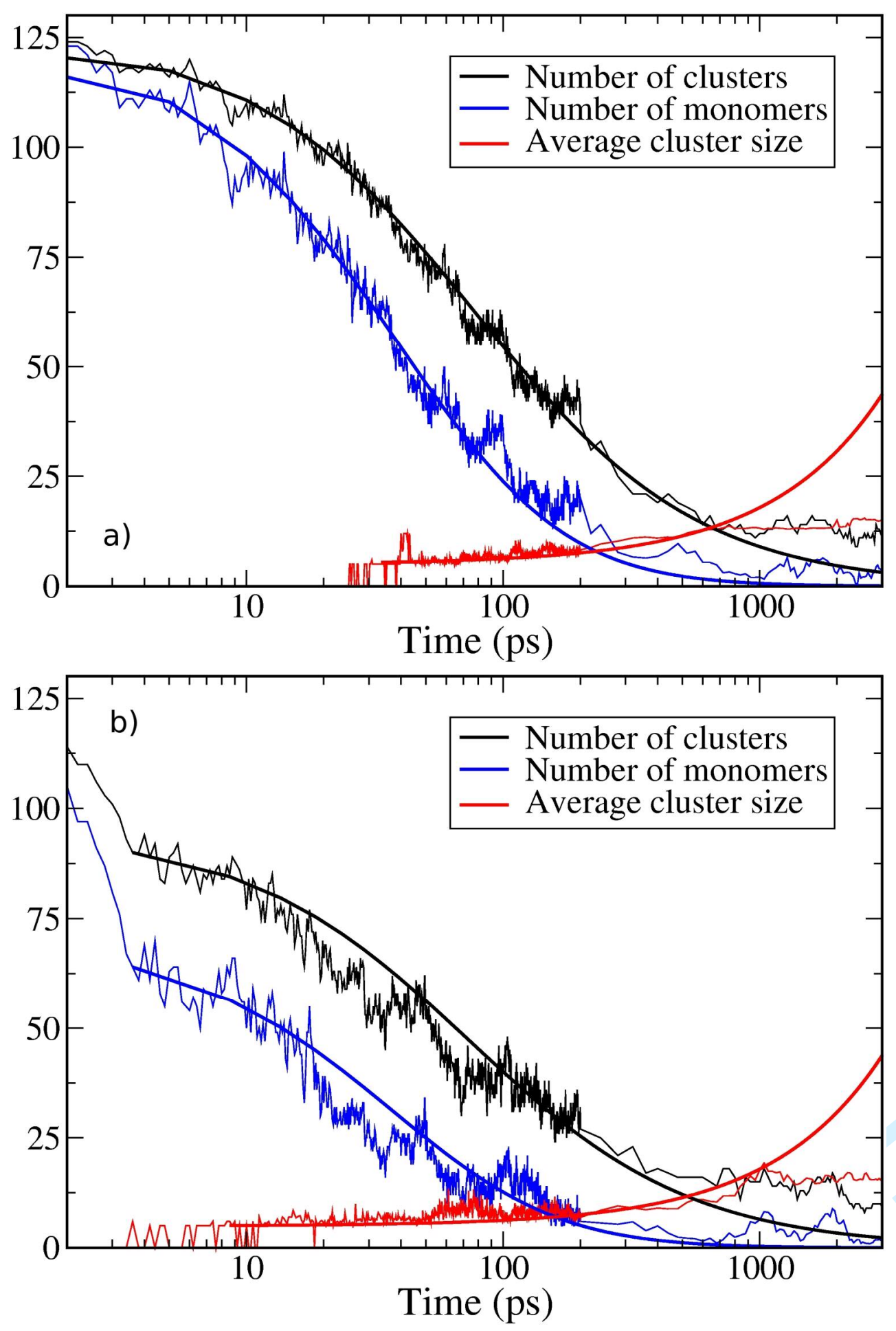

Figure 5. Comparison between simulation results (thin lines) and kinetic theory predictions (smooth thick lines) for the time evolution of the surfactant aggregation process, using: a) the united-atom model; b) the all-atom model. 

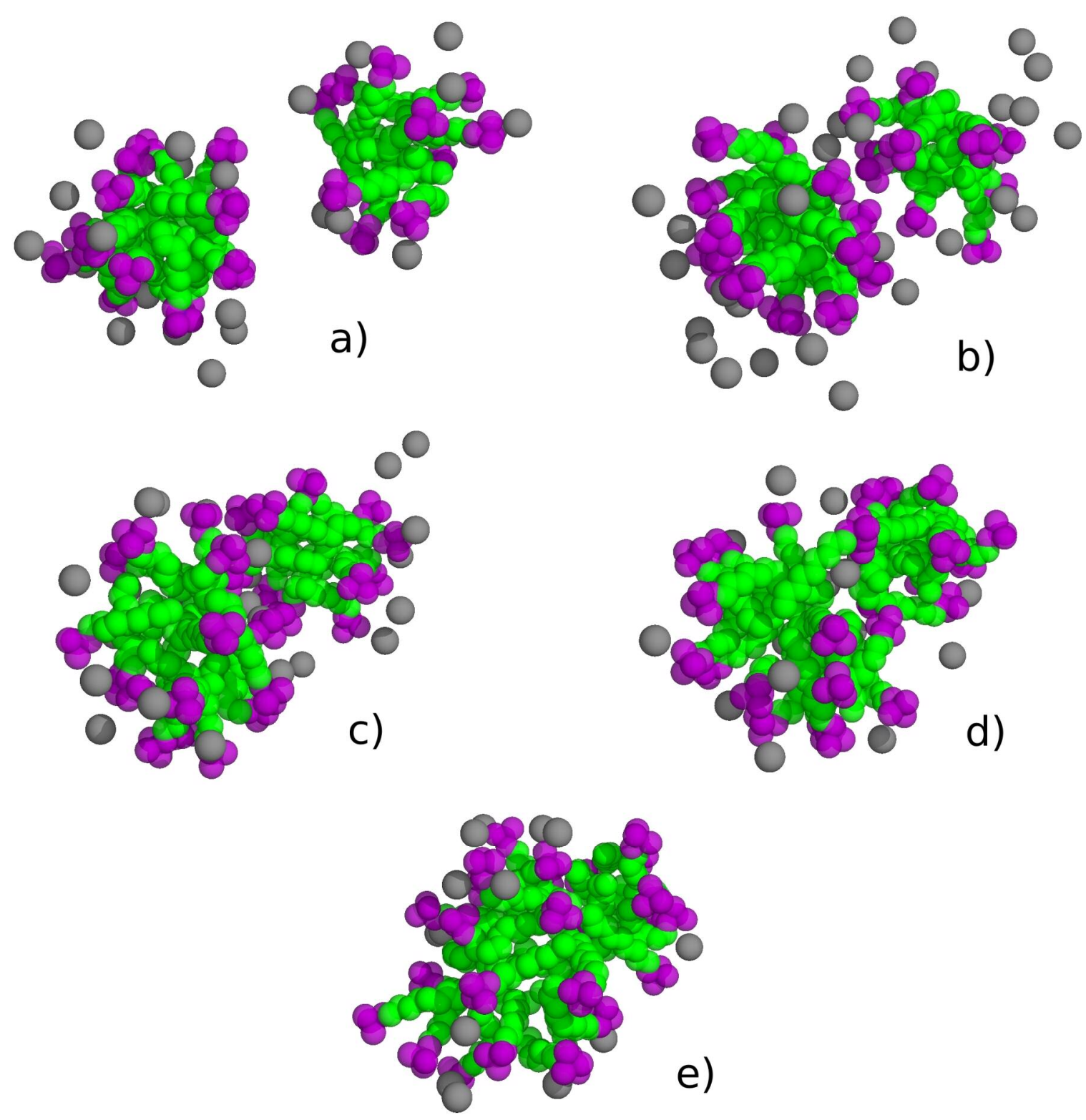

Figure 6. Snapshots of the united-atom simulation showing the fusion of two micelles. Simulation times are: a) 2180 ps; b) 4260 ps; c) 4300 ps; d) 4360 ps; e) 4600 ps. Color coding is the same as in Figure 4. Water molecules have been omitted for clarity. 

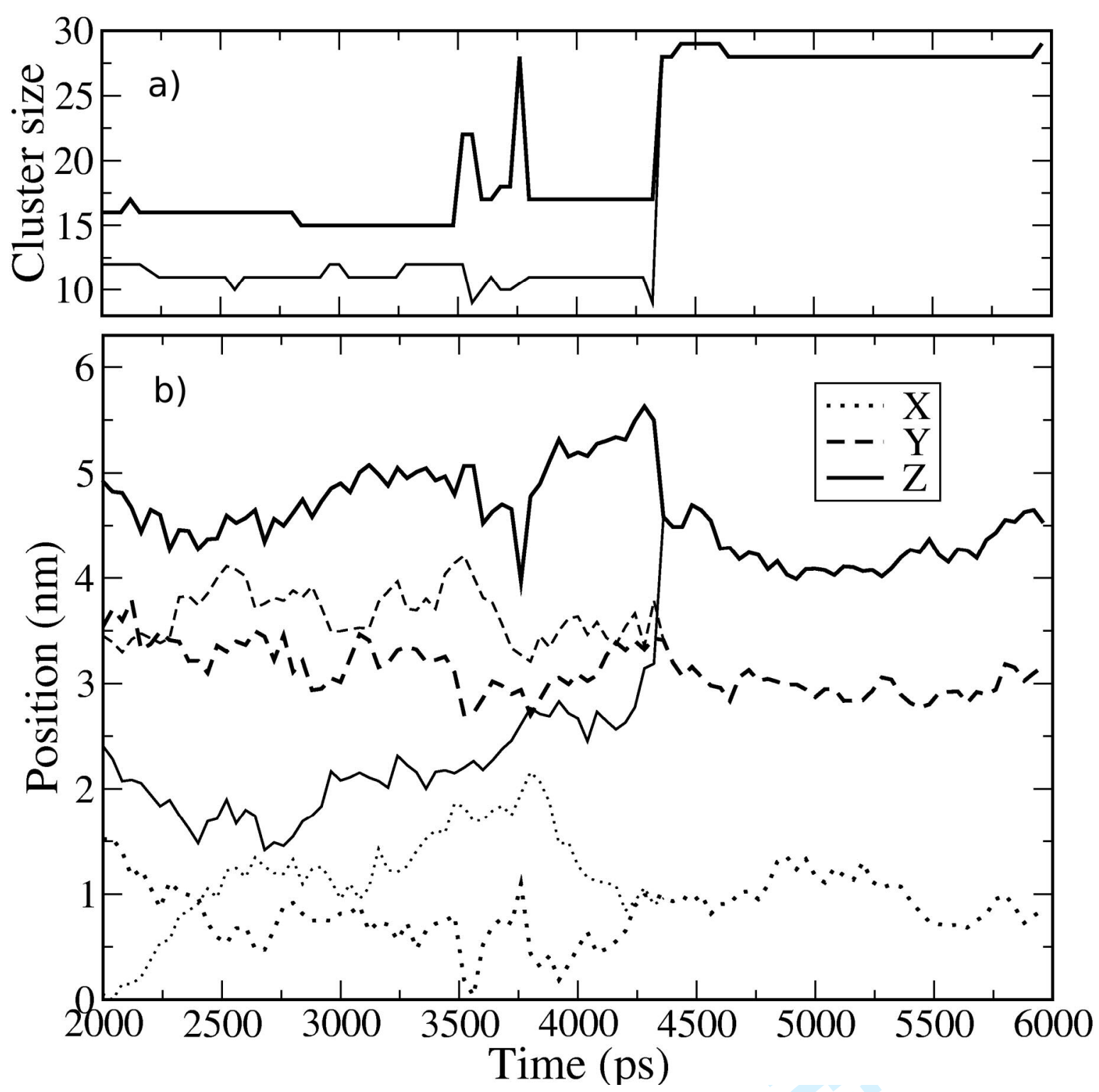

Figure 7. Plot showing the fusion of two micelles, corresponding to the snapshots of Figure 6. Panel a) shows the evolution of the size of both micelles, while panel b) plots the $x, y$ and $z$ coordinates of the center-of-mass of both micelles. Thin lines are for the smaller micelle (on the right in Figure 6) and thick lines are for the larger micelle (on the left in Figure 6). The fusion takes place at $4360 \mathrm{ps}$. 


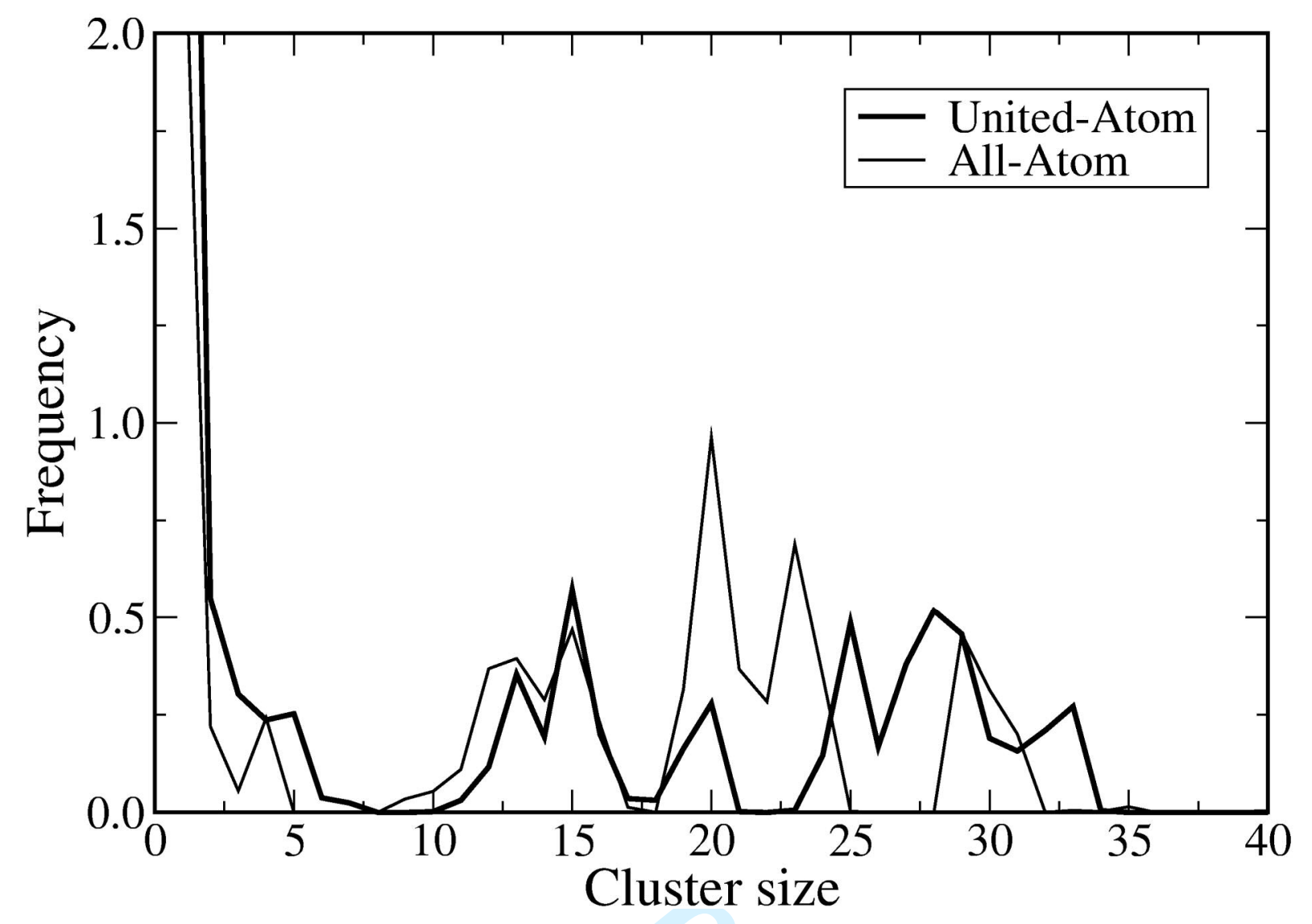

Figure 8. Cluster size distributions for the united-atom (thick line) and all-atom (thin line) models, averaged over the last $7 \mathrm{~ns}$ of simulation time. 


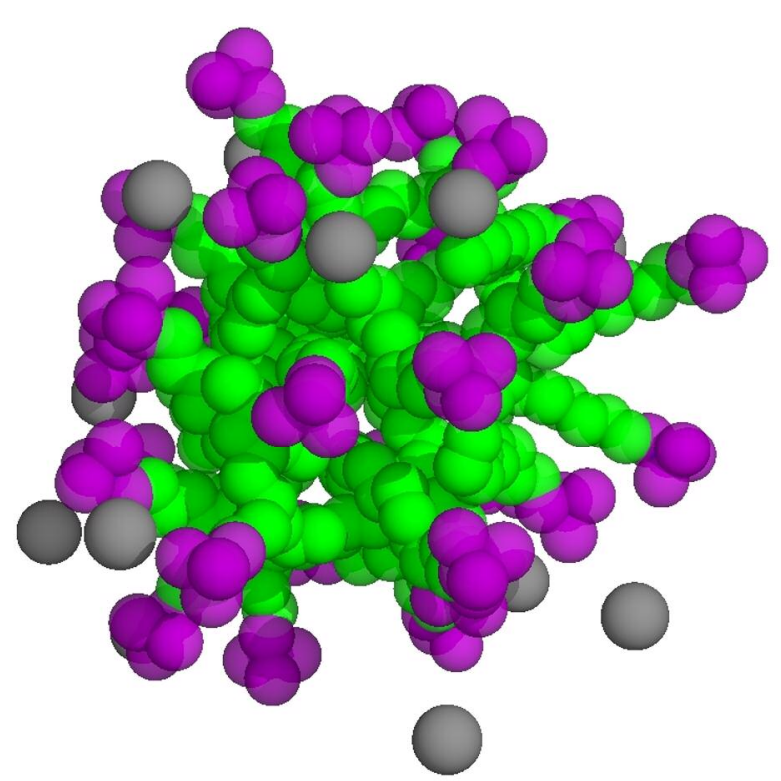

a)

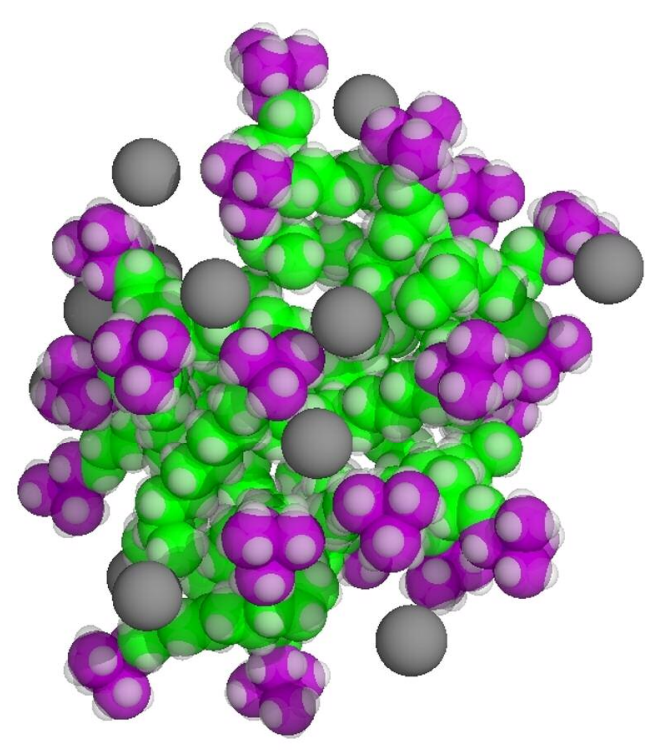

b)

Figure 9. Snapshots of typical micelles obtained during the simulation runs with the united-atom (a) and all-atom (b) models. Color coding is the same as in Figure 4 and water molecules have been omitted for clarity. 

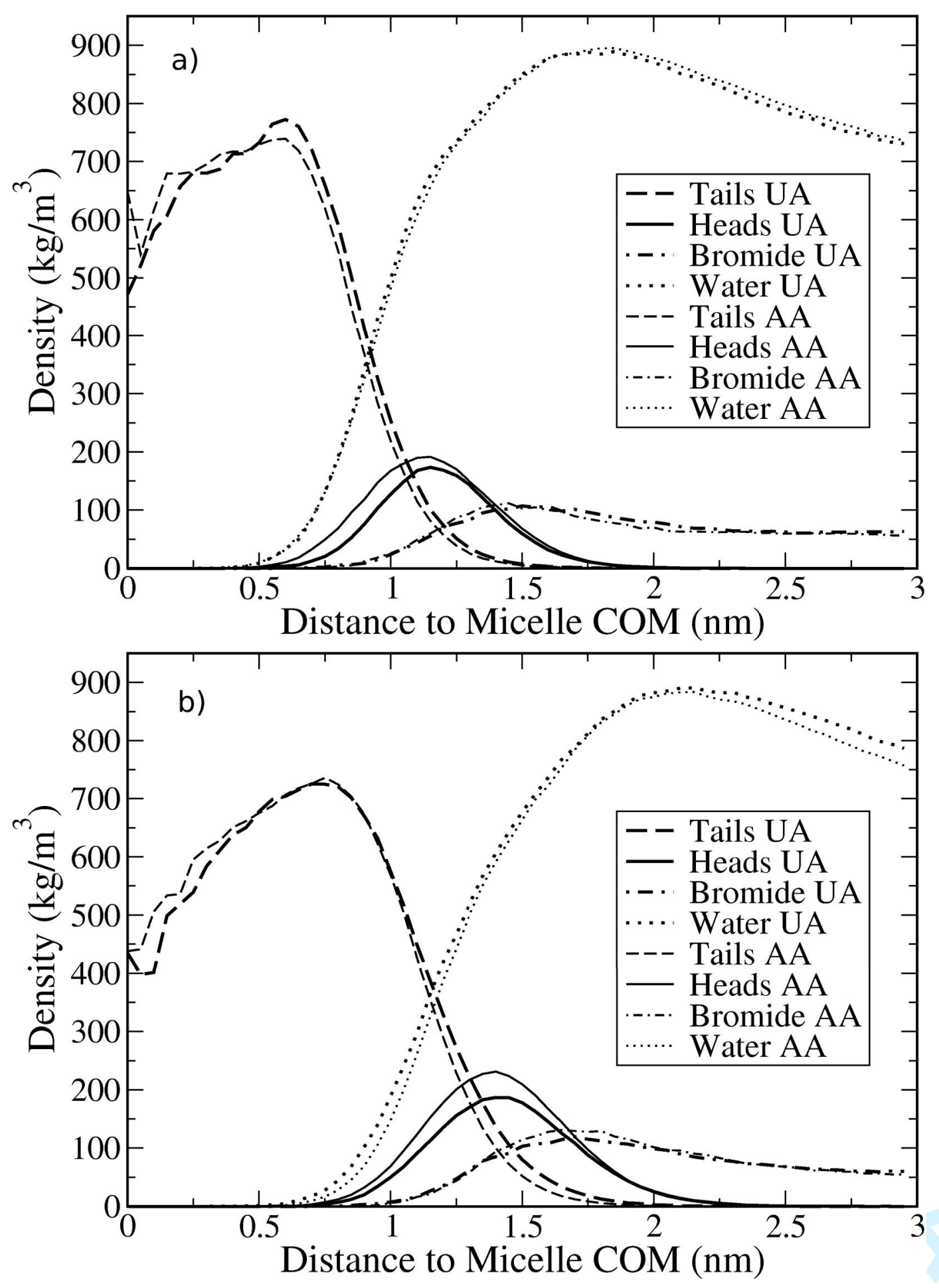

Figure 10. Comparison between the density profiles of micelles obtained using the united-atom (thick lines) and all-atom (thin lines) models. Panel a) shows the profiles for micelles containing 15 or 16 surfactant molecules, while panel b) is for micelles with 29 or 30 surfactants. 


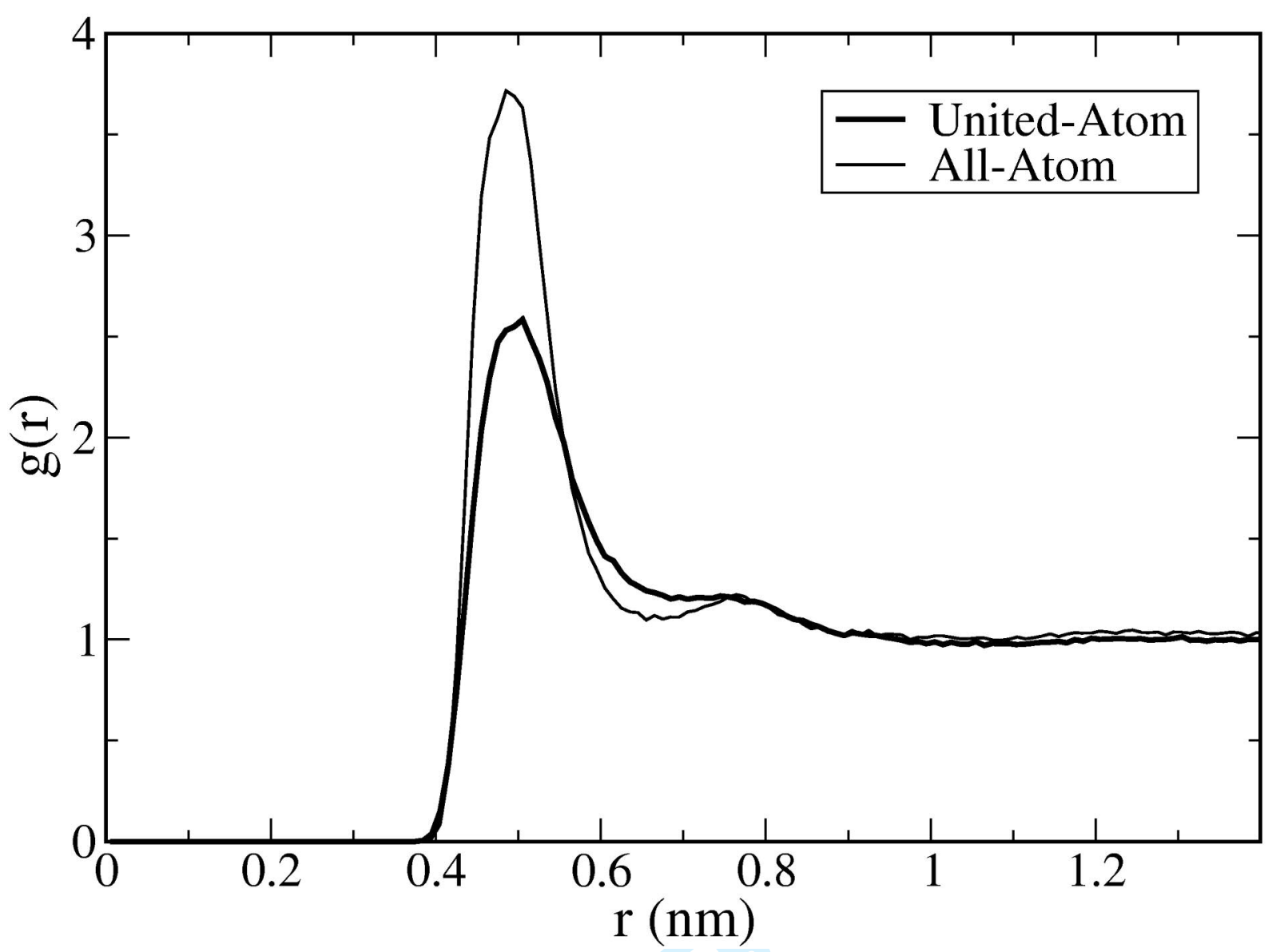

Figure 11. Radial distribution functions between surfactant nitrogen atoms and bromide counterions for the united-atom (thick line) and all-atom (thin line) models. 


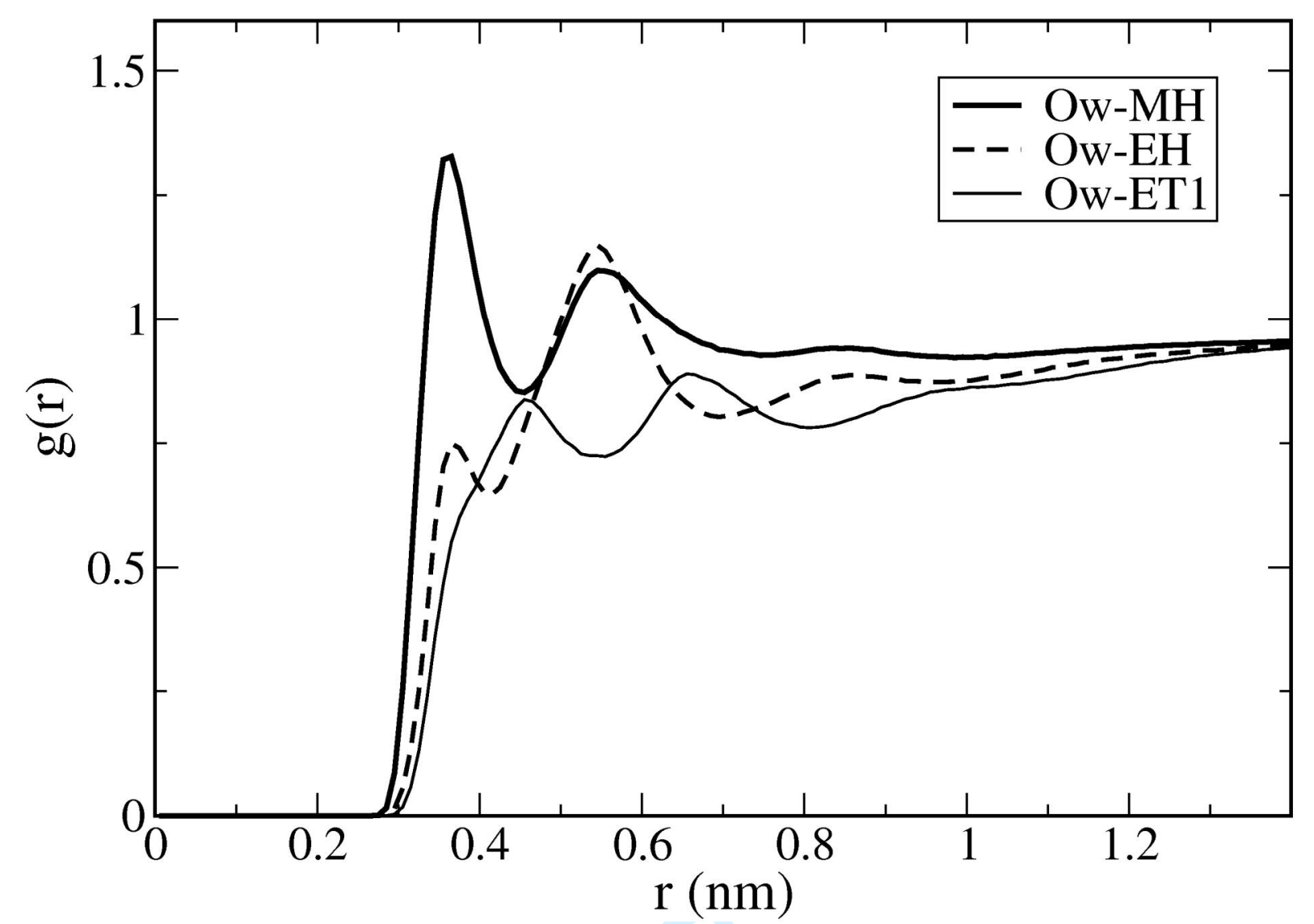

Figure 12. Radial distribution functions, for the all-atom model, between water oxygen atoms and carbon atoms in the surfactant molecule: $\mathrm{MH}$ outer head carbons (thick line); EH inner head carbon (dashed line); ET1 first tail carbon (thin line). 


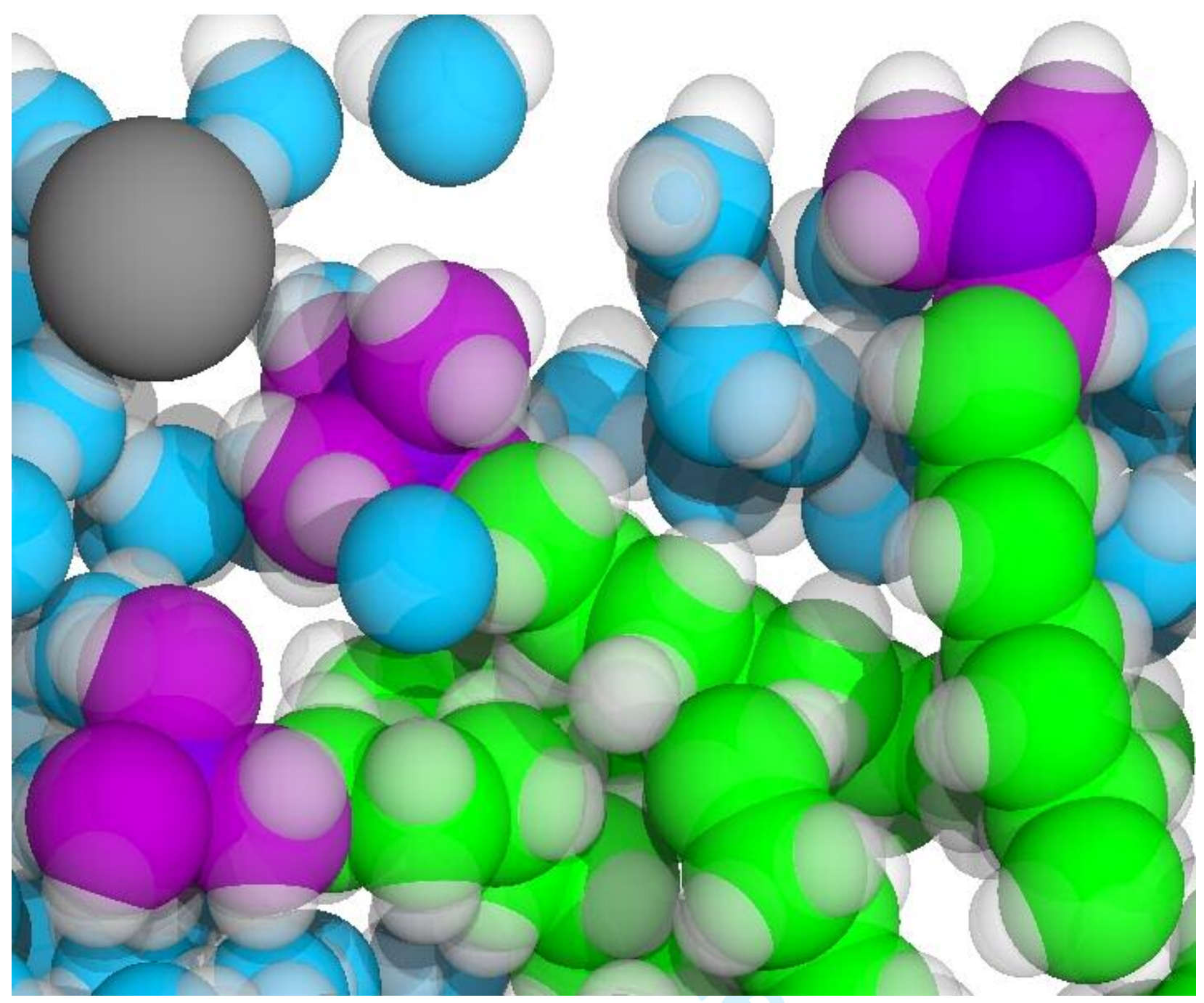

Figure 13. Snapshot of a cross-section of the simulation box in the AA run. The snapshot is blown up to show the structure of a typical micelle in detail. Color coding is the same as in Figure 4, except for water oxygens, which are represented by blue spheres. 


\section{For Table of Contents Use Only}

TOC_GRAPHIC

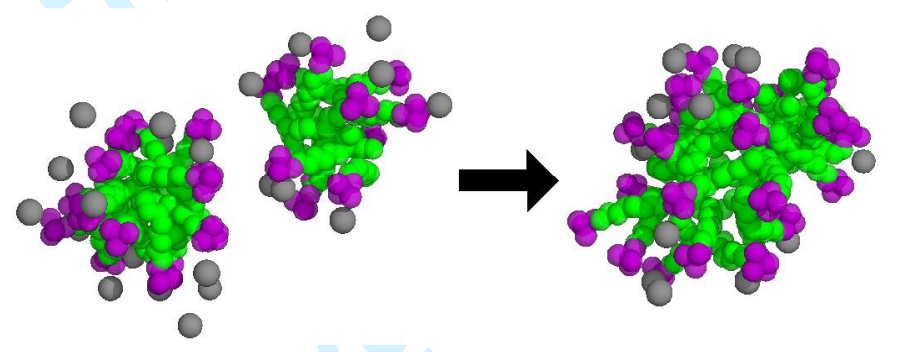

